ESAIM: M2AN 49 (2015) 503-528

DOI: $10.1051 / \mathrm{m} 2 \mathrm{an} / 2014046$
ESAIM: Mathematical Modelling and Numerical Analysis

www.esaim-m2an.org

\title{
A NITSCHE FINITE ELEMENT METHOD FOR DYNAMIC CONTACT: 2. STABILITY OF THE SCHEMES AND NUMERICAL EXPERIMENTS
}

\author{
Franz Chouly ${ }^{1}$, Patrick Hild ${ }^{2}$ and Yves Renard 3
}

\begin{abstract}
In a previous paper [F. Chouly, P. Hild and Y. Renard, A Nitsche finite element method for dynamic contact. 1. Space semi-discretization and time-marching schemes. ESAIM: M2AN 49 (2015) 481-502.], we adapted Nitsche's method to the approximation of the linear elastodynamic unilateral contact problem. The space semi-discrete problem was analyzed and some schemes ( $\theta$-scheme, Newmark and a new hybrid scheme) were proposed and proved to be well-posed under appropriate CFL conditions. In the present paper we look at the stability properties of the above-mentioned schemes and we proceed to the corresponding numerical experiments. In particular we prove and illustrate numerically some interesting stability and (almost) energy conservation properties of Nitsche's semi-discretization combined to the new hybrid scheme.
\end{abstract}

Mathematics Subject Classification. 65N12, 65N30, 74M15.

Received March 13, 2014. Revised September 1st, 2014.

Published online 17 March 2015.

\section{INTRODUCTION AND PROBLEM SETTING}

In considering contact problems for elastodynamics, it is well-known that special difficulties arise when semidiscretization in space is carried out with the standard finite element method ("standard" refers to a mixed method with a Lagrange multiplier standing for the contact stress). Namely the semi-discrete problem is illposed (see, e.g., [19]), and, additionally, when conservative time-marching schemes like Crank-Nicolson are applied, the resulting numerical solution exhibits (see, e.g., $[10,19,20])$ :

1. some spurious oscillations on the contact stress that quickly contaminate the whole solution (velocity and displacement);

2. a poor energetic behaviour, with a mechanical energy increasing at each impact instead of remaining constant (for a conservative system).

Note that these problems are not cured if the time-step is chosen smaller, and are even amplified. One obvious solution is to resort to a dissipative time-marching scheme like backward Euler, but to the price that the

Keywords and phrases. Unilateral contact, elastodynamics, Nitsche's method, time-marching schemes, stability.

1 Laboratoire de Mathématiques de Besançon - UMR CNRS 6623, Université de Franche Comté, 16 route de Gray, 25030 Besançon cedex, France. franz.chouly@univ-fcomte.fr

2 Institut de Mathématiques de Toulouse - UMR CNRS 5219, Université Paul Sabatier, 118 route de Narbonne, 31062 Toulouse cedex 9, France. patrick.hild@math.univ-toulouse.fr

3 Université de Lyon, CNRS, INSA-Lyon, ICJ UMR5208, LaMCoS UMR5259, 69621 Villeurbanne, France. yves.renard@insa-lyon.fr 
displacement is quickly damped, after a few impacts (see Prop. 3.2 and Sect. 4.1.2.a). So various strategies have been proposed to treat these severe numerical pathologies and to obtain stable and conservative schemes, without compromising the computational efficiency and the simplicity of implementation (see the state-of-the-art and some comparisons in, e.g., $[10,19,20])$. We may summarize the existing works as follows:

1. an impact law can be added, as for rigid bodies impact [23]. Unfortunately this does not remove all the spurious oscillations. Moreover, for deformable bodies, such an impact law should be unnecessary;

2. the contact term can be discretized in an implicit fashion, as in $[4,9,11,17]$ or as in $[24,25]$ (semi-explicit schemes). The drawback of this approach is mostly energy dissipation when contact conditions are activated, so that the shape of the solution is damped and changes after a few impacts (see e.g., numerical tests in [10]).

3 . the time-discretization is modified to preserve the energy. This can be done for instance through the "velocity update method" [22], where an extra correction term is added each time the velocity is updated. An alternative strategy consists in using the contact condition in velocity, to obtain energy conservation [21] (see also [3] for viscoelastodynamic contact). In the context of penalty methods, a very specific time-discretization of the penalty term allows to conserve a modified discrete energy $[2,16]$. Generally all these schemes do not prevent spurious oscillations on the contact stress, and do not fulfill exactly the contact constraint [26].

4. the space semi-discretization is changed, so that the contact nodes do not work, which leads to the socalled "modified mass" method [19] and its extensions $[14,15,26]$. This strategy leads in particular to a well-posed semi-discrete problem. Moreover it is energy conservative, and the numerical solution is almost free of spurious oscillations. Its main drawback is that the mass matrix needs to be changed, but this can be done efficiently and without affecting too much the numerical solution.

When applied to contact-impact in elastodynamics, Nitsche's method has the good property that it leads to a well-posed semi-discrete problem (system of Lipschitz differential equations) as it is shown in [5]. This feature is shared by the penalty method and modified mass methods too. Moreover the symmetric variant of Nitsche's space semi-discretization conserves an augmented energy, like the penalty method (see, [5]). On the contrary a standard finite element discretization using Lagrange multipliers leads to an ill-posed measure differential inclusion (see, e.g., [19]).

In this second part, we study theoretically the stability and energy conservation properties of fully discrete schemes based on space semi-discretization with Nitsche's method. For the $\theta$-scheme and the Newmark scheme, combined to the symmetric variant of Nitsche, the unconditional stability can be proved only for the most dissipative schemes $\left(\theta=1\right.$ for the $\theta$-scheme (Cor. 3.2) and $\gamma=1, \beta=\frac{1}{2}$ for Newmark (Cor. 3.5)). Conversely, for the new hybrid scheme, there is no such limitation, and unconditional stability is recovered, even though small numerical dissipation occurs during the contact phase (Prop. 3.7).

We complete this theoretical work with numerical experiments, to illustrate the established stability and energy conservation properties, and to study as well the presence of spurious oscillations on the displacement and the contact stress.

Let us introduce some useful notations. In what follows, bold letters like $\mathbf{u}, \mathbf{v}$, indicate vector or tensor valued quantities, while the capital ones (e.g., $\mathbf{V}, \mathbf{K} \ldots$...) represent functional sets involving vector fields. As usual, we denote by $\left(H^{s}(.)\right)^{d}, s \in \mathbb{R}, d=1,2,3$ the Sobolev spaces in one, two or three space dimensions (see [1]). The usual norm of $\left(H^{s}(D)\right)^{d}$ is denoted by $\|\cdot\|_{s, D}$ and we keep the same notation when $d=1$ or $d>1$. The letter $C$ stands for a generic constant, independent of the discretization parameters.

We consider an elastic body $\Omega$ in $\mathbb{R}^{d}$ with $d=1,2,3$. Small strain assumptions are made (as well as plane strain when $d=2)$. The boundary $\partial \Omega$ of $\Omega$ is polygonal $(d=2)$ or polyhedral $(d=3)$. The normal unit outward vector on $\partial \Omega$ is denoted $\mathbf{n}$. We suppose that $\partial \Omega$ consists in three nonoverlapping parts $\Gamma_{D}, \Gamma_{N}$ and the contact boundary $\Gamma_{C}$, with meas $\left(\Gamma_{D}\right)>0$ and meas $\left(\Gamma_{C}\right)>0$. The contact boundary is supposed to be a straight line segment when $d=2$ or a polygon when $d=3$ to simplify. In the reference configuration, the body is in contact on $\Gamma_{C}$ with a rigid foundation and we suppose that the unknown contact zone during deformation is included in $\Gamma_{C}$. The body is clamped on $\Gamma_{D}$ for the sake of simplicity. It is subjected to volume forces $\mathbf{f}$ in $\Omega$ and to surface loads $\mathrm{g}$ on $\Gamma_{N}$. 
We consider the unilateral contact problem in linear elastodynamics during a period of time $[0, T)$ where $T>0$ is the final time. We denote by $\Omega_{T}:=(0, T) \times \Omega$ the time-space domain, and similarly $\Gamma_{D T}:=(0, T) \times \Gamma_{D}$, $\Gamma_{N T}:=(0, T) \times \Gamma_{N}$ and $\Gamma_{C T}:=(0, T) \times \Gamma_{C}$. The problem then consists in finding the displacement field $\mathbf{u}:[0, T) \times \Omega \rightarrow \mathbb{R}^{d}$ verifying the equations and conditions (1.1)-(1.2):

$$
\begin{aligned}
& \rho \ddot{\mathbf{u}}-\operatorname{div} \boldsymbol{\sigma}(\mathbf{u})=\mathbf{f}, \quad \boldsymbol{\sigma}(\mathbf{u})=\mathbf{A} \varepsilon(\mathbf{u}) \quad \text { in } \Omega_{T}, \\
& \mathbf{u}=\mathbf{0} \quad \text { on } \Gamma_{D T}, \\
& \boldsymbol{\sigma}(\mathbf{u}) \mathbf{n}=\mathbf{g} \quad \text { on } \Gamma_{N T}, \\
& \mathbf{u}(0, \cdot)=\mathbf{u}_{0} \quad \dot{\mathbf{u}}(0, \cdot)=\dot{\mathbf{u}}_{0} \quad \text { in } \Omega,
\end{aligned}
$$

where the notation $\dot{\mathbf{x}}$ is used for the time-derivative of a vector field $\mathbf{x}$ on $\Omega_{T}$, so that $\dot{\mathbf{u}}$ is the velocity of the elastic body and $\ddot{\mathbf{u}}$ its acceleration; $\mathbf{u}_{0}$ and $\dot{\mathbf{u}}_{0}$ being the initial displacement and the velocity. The density of the elastic material denoted by $\rho$ is supposed to be constant to simplify (this is not restrictive and the results can be extended straightforwardly for a variable density). The notation $\boldsymbol{\sigma}=\left(\sigma_{i j}\right), 1 \leq i, j \leq d$, stands for the stress tensor field and div denotes the divergence operator of tensor valued functions. The notation $\varepsilon(\mathbf{v})=\left(\nabla \mathbf{v}+\nabla \mathbf{v}^{T}\right) / 2$ represents the linearized strain tensor field and $\mathbf{A}$ is the fourth order symmetric elasticity tensor having the usual uniform ellipticity and boundedness property. For any displacement field $\mathbf{v}$ and for any density of surface forces $\boldsymbol{\sigma}(\mathbf{v}) \mathbf{n}$ defined on $\partial \Omega$ we adopt the following notation

$$
\mathbf{v}=v_{n} \mathbf{n}+\mathbf{v}_{\mathbf{t}} \quad \text { and } \quad \boldsymbol{\sigma}(\mathbf{v}) \mathbf{n}=\sigma_{n}(\mathbf{v}) \mathbf{n}+\boldsymbol{\sigma}_{\mathbf{t}}(\mathbf{v}),
$$

where $\mathbf{v}_{\mathbf{t}}\left(\right.$ resp. $\left.\boldsymbol{\sigma}_{\mathbf{t}}(\mathbf{v})\right)$ is the tangential component of $\mathbf{v}($ resp. $\boldsymbol{\sigma}(\mathbf{v}) \mathbf{n})$. The conditions describing unilateral contact without friction on $\Gamma_{C T}$ are:

$$
u_{n} \leq 0 \quad \sigma_{n}(\mathbf{u}) \leq 0 \quad \sigma_{n}(\mathbf{u}) u_{n}=0 \quad \sigma_{\mathbf{t}}(\mathbf{u})=0 .
$$

The rest of the paper is outlined as follows. In Section 2, the different fully discrete formulations for dynamic contact with Nitsche are re-introduced. Energy estimates for each time-marching scheme are presented in Section 3. Numerical experiments are described in Section 4. Conclusions are drawn and perspectives are given in Section 5.

\section{The Nitsche-BASed finite Element Method}

\subsection{Definitions and notations}

Let us introduce $\mathbf{V}:=\left\{\mathbf{v} \in\left(H^{1}(\Omega)\right)^{d}: \mathbf{v}=\mathbf{0}\right.$ on $\left.\Gamma_{D}\right\}$ and let $\mathbf{V}^{h} \subset \mathbf{V}$ be a family of finite element spaces (see [6]) indexed by $h$ coming from a family $\mathcal{T}^{h}$ of triangulations of the domain $\Omega\left(h=\max _{K \in \mathcal{T}^{h}} h_{K}\right.$ where $h_{K}$ is the diameter of the triangle $K$ ). The family of triangulations is supposed regular and conformal to the subdivision of the boundary into $\Gamma_{D}, \Gamma_{N}$ and $\Gamma_{C}$. To fix ideas, we choose a standard Lagrange finite element method of degree $k$ with $k=1$ or $k=2$, i.e.:

$$
\mathbf{V}^{h}=\left\{\mathbf{v}^{h} \in\left(\mathscr{C}^{0}(\bar{\Omega})\right)^{d}: \mathbf{v}^{h}{ }_{K} \in\left(P_{k}(K)\right)^{d}, \forall K \in \mathcal{T}^{h}, \mathbf{v}^{h}=\mathbf{0} \text { on } \Gamma_{D}\right\} .
$$

We introduce the notation $[\cdot]_{+}$(resp. $\left.[\cdot]_{-}\right)$for the positive (resp. negative) part of a scalar quantity $a \in \mathbb{R}:$ $[a]_{+}=a$ if $a>0$, and $[a]_{+}=0$ otherwise. Moreover

$$
a=[a]_{+}-[a]_{-}
$$

for all $a \in \mathbb{R}$. The positive part has the following properties for all $a, b \in \mathbb{R}$ :

$$
\begin{aligned}
a \leq[a]_{+}, a[a]_{+} & =[a]_{+}^{2}, \\
\left([a]_{+}-[b]_{+}\right)(a-b) & \geq\left([a]_{+}-[b]_{+}\right)^{2} \geq 0 .
\end{aligned}
$$


The Heaviside function will be noted $H(\cdot)$. We define it as follows, for $a \in \mathbb{R}$ (with a slight modification when $a=0$ so that $H(a)+H(-a)=1, \forall a \in \mathbb{R})$ :

$$
H(a)= \begin{cases}1 & \text { if } a>0 \\ \frac{1}{2} & \text { if } a=0, \\ 0 & \text { if } a<0\end{cases}
$$

We consider in what follows that $\gamma=\gamma_{h}$ is a positive piecewise constant function on the contact interface $\Gamma_{C}$ which satisfies

$$
\left.\gamma_{h}\right|_{K \cap \Gamma_{C}}=\gamma_{0} h_{K}
$$

for every $K$ that has a non-empty intersection of dimension $d-1$ with $\Gamma_{C}$, and where $\gamma_{0}$ is a given positive constant. Note that the value of $\gamma_{h}$ on element intersections has no influence.

We introduce the discrete linear operator

$$
P_{\gamma_{h}}: \begin{aligned}
& \mathbf{V}^{h} \\
& \mathbf{v}^{h} \mapsto v_{n}^{h}-\gamma_{h} \sigma_{n}\left(\Gamma_{C}\right)
\end{aligned}
$$

and also the bilinear form:

$$
A_{\Theta \gamma_{h}}\left(\mathbf{u}^{h}, \mathbf{v}^{h}\right)=a\left(\mathbf{u}^{h}, \mathbf{v}^{h}\right)-\int_{\Gamma_{C}} \Theta \gamma_{h} \sigma_{n}\left(\mathbf{u}^{h}\right) \sigma_{n}\left(\mathbf{v}^{h}\right) \mathrm{d} \Gamma,
$$

where $\Theta \in \mathbb{R}$ is a fixed parameter. Our space semi-discretized Nitsche-based method for unilateral contact problems in elastodynamics then reads:

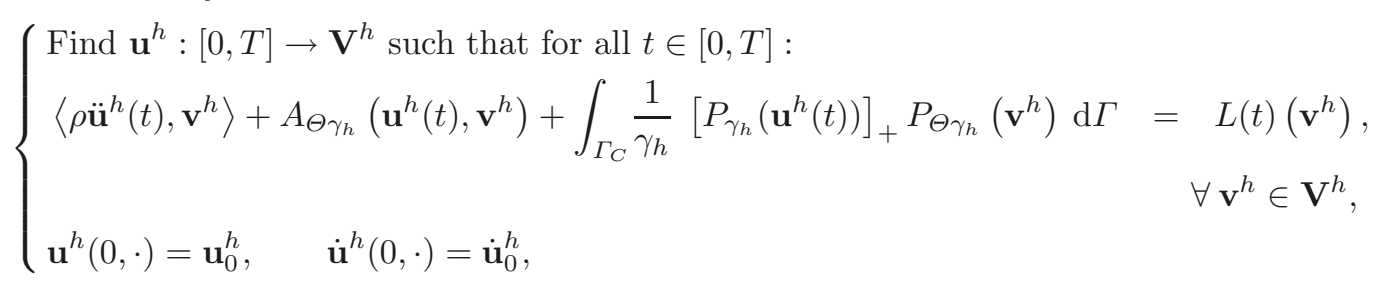

with $P_{\Theta \gamma_{h}}\left(\mathbf{v}_{h}\right):=v_{n}^{h}-\Theta \gamma_{h} \sigma_{n}\left(\mathbf{v}^{h}\right)$ and where $\mathbf{u}_{0}^{h}\left(\right.$ resp. $\left.\dot{\mathbf{u}}_{0}^{h}\right)$ is an approximation in $\mathbf{V}^{h}$ of the initial displacement $\mathbf{u}_{0}$ (resp. the initial velocity $\dot{\mathbf{u}}_{0}$ ), for instance the Lagrange interpolant or the $L^{2}(\Omega)$-projection of $\mathbf{u}_{0}$ (resp. $\left.\dot{\mathbf{u}}_{0}\right)$. The notation $\langle\cdot, \cdot\rangle$ stands for the $L^{2}(\Omega)$-inner product.

In the rest of the paper, $\tau>0$ denotes the time-step, and we consider a uniform discretization of the time interval $[0, T]:\left(t^{0}, \ldots, t^{N}\right)$, with $t^{n}=n \tau, n=0, \ldots, N$. Let $\theta \in[0,1]$, we use the notation:

$$
\mathbf{x}^{h, n+\theta}=(1-\theta) \mathbf{x}^{h, n}+\theta \mathbf{x}^{h, n+1}
$$

for arbitrary quantities $\mathbf{x}^{h, n}, \mathbf{x}^{h, n+1} \in \mathbf{V}^{h}$. Hereafter we denote by $\mathbf{u}^{h, n}$ (resp. $\dot{\mathbf{u}}^{h, n}$ and $\ddot{\mathbf{u}}^{h, n}$ ) the resulting discretized displacement (resp. velocity and acceleration) at time step $t^{n}$.

\subsection{Proposed time-marching schemes}

\subsubsection{A $\theta$-scheme}

We approximate the semi-discrete problem (2.5) using a $\theta$-scheme, whose parameter is $\theta \in[0,1]$. For $n \geq 0$, the fully discretized problem reads:

$$
\left\{\begin{array}{l}
\text { Find } \mathbf{u}^{h, n+1}, \dot{\mathbf{u}}^{h, n+1}, \ddot{\mathbf{u}}^{h, n+1} \in \mathbf{V}^{h} \text { such that: } \\
\mathbf{u}^{h, n+1}=\mathbf{u}^{h, n}+\tau \dot{\mathbf{u}}^{h, n+\theta} \\
\dot{\mathbf{u}}^{h, n+1}=\dot{\mathbf{u}}^{h, n}+\tau \ddot{\mathbf{u}}^{h, n+\theta} \\
\left\langle\rho \ddot{\mathbf{u}}^{h, n+1}, \mathbf{v}^{h}\right\rangle+A_{\Theta \gamma_{h}}\left(\mathbf{u}^{h, n+1}, \mathbf{v}^{h}\right)+\int_{\Gamma_{C}} \frac{1}{\gamma_{h}}\left[P_{\gamma_{h}}\left(\mathbf{u}^{h, n+1}\right)\right]_{+} P_{\Theta \gamma_{h}}\left(\mathbf{v}^{h}\right) \mathrm{d} \Gamma=L^{n+1}\left(\mathbf{v}^{h}\right), \\
\quad \forall \mathbf{v}^{h} \in \mathbf{V}^{h}
\end{array}\right.
$$


with initial conditions $\mathbf{u}^{h, 0}=\mathbf{u}_{0}^{h}, \dot{\mathbf{u}}^{h, 0}=\dot{\mathbf{u}}_{0}^{h}, \ddot{\mathbf{u}}^{h, 0}=\ddot{\mathbf{u}}_{0}^{h}$ (see Rem. 2.1 below), and where $L^{n+1}(\cdot)=L\left(t^{n+1}\right)(\cdot)$.

We recall that this scheme is consistent of order 1 in $\tau$ if $\theta \neq \frac{1}{2}$ and of order 2 if $\theta=\frac{1}{2}$. For linear elastodynamics, it is also known to be unconditionally stable for $\theta \geq \frac{1}{2}$ and conditionally stable when $\theta<\frac{1}{2}$. It is fully explicit when $\theta=0$. It is dissipative when $\theta>\frac{1}{2}$ and conserves the energy when $\theta=\frac{1}{2}$.

Remark 2.1. The initial condition $\ddot{\mathbf{u}}^{h, 0}$ is determined in fact through:

$$
\left\langle\rho \ddot{\mathbf{u}}_{0}^{h}, \mathbf{v}^{h}\right\rangle=L^{0}\left(\mathbf{v}^{h}\right)-A_{\Theta \gamma_{h}}\left(\mathbf{u}_{0}^{h}, \mathbf{v}^{h}\right)-\int_{\Gamma_{C}} \frac{1}{\gamma_{h}}\left[P_{\gamma_{h}}\left(\mathbf{u}_{0}^{h}\right)\right]_{+} P_{\Theta \gamma_{h}}\left(\mathbf{v}^{h}\right) \mathrm{d} \Gamma \quad \forall \mathbf{v}^{h} \in \mathbf{V}^{h},
$$

which corresponds to inverting the mass matrix $\mathbf{M}^{h}$.

\subsubsection{A Newmark scheme}

We approximate the semi-discrete problem (2.5) with a Newmark scheme, whose parameters are $\beta \in[0,1 / 2]$, $\gamma \in[0,1]$. For $n \geq 0$, the fully discretized problem reads:

$$
\left\{\begin{array}{l}
\text { Find } \mathbf{u}^{h, n+1}, \dot{\mathbf{u}}^{h, n+1}, \ddot{\mathbf{u}}^{h, n+1} \in \mathbf{V}^{h} \text { such that: } \\
\mathbf{u}^{h, n+1}=\mathbf{u}^{h, n}+\tau \dot{\mathbf{u}}^{h, n}+\frac{\tau^{2}}{2} \ddot{\mathbf{u}}^{h, n+2 \beta}, \\
\dot{\mathbf{u}}^{h, n+1}=\dot{\mathbf{u}}^{h, n}+\tau \ddot{\mathbf{u}}^{h, n+\gamma} \\
\left\langle\rho \ddot{\mathbf{u}}^{h, n+1}, \mathbf{v}^{h}\right\rangle+A_{\Theta \gamma_{h}}\left(\mathbf{u}^{h, n+1}, \mathbf{v}^{h}\right)+\int_{\Gamma_{C}} \frac{1}{\gamma_{h}}\left[P_{\gamma_{h}}\left(\mathbf{u}^{h, n+1}\right)\right]_{+} P_{\Theta \gamma_{h}}\left(\mathbf{v}^{h}\right) \mathrm{d} \Gamma=L^{n+1}\left(\mathbf{v}^{h}\right), \\
\quad \forall \mathbf{v}^{h} \in \mathbf{V}^{h}
\end{array}\right.
$$

with initial conditions $\mathbf{u}^{h, 0}=\mathbf{u}_{0}^{h}, \dot{\mathbf{u}}^{h, 0}=\dot{\mathbf{u}}_{0}^{h}, \ddot{\mathbf{u}}^{h, 0}=\ddot{\mathbf{u}}_{0}^{h}$ (see Rem. 2.1).

This scheme is consistent of order 1 in $\tau$ when $\gamma \neq \frac{1}{2}$, of order 2 when $\gamma=\frac{1}{2}$ and $\beta \neq \frac{1}{12}$, and of order 4 when $\gamma=\frac{1}{2}$ and $\beta=\frac{1}{12}$. When applied to linear elastodynamics (without contact), it is not stable when $\gamma<\frac{1}{2}$, unconditionally stable when $\gamma \geq \frac{1}{2}$ and $\frac{\gamma}{2} \leq \beta \leq \frac{1}{2}$, and conditionally stable when $\gamma \geq \frac{1}{2}$ and $0 \leq \beta \leq \frac{\gamma}{2}$.

\subsubsection{A new Hybrid scheme}

We introduce a new time-marching scheme for problem (2.5). Inspired by the works of Gonzalez [13] and Hauret and Le Tallec [16], the idea is to propose an hybrid discretization of the Nitsche-based contact term: the linear part of problem (2.5) is treated with a conservative Crank-Nicolson scheme, whereas the non-linear part arising from contact is discretized with a linear combination of Crank-Nicolson and Midpoint schemes. The interest of such a strategy is that the resulting scheme is unconditionally stable in the symmetric case (see Prop. 3.7) and still consistent of second order in time. Conversely to the method proposed in [16] for penalty, it is no more conservative strictly speaking. Yet the diffusive effects are very small and only occur during the contact/non-contact transition (see the proof of Prop. 3.7). Moreover, this new scheme is easy to implement, and only requires slight modifications from the standard Crank-Nicolson implementations. It could be adapted for penalty as well. For $n \geq 0$, the fully discretized problem reads:

$$
\left\{\begin{array}{l}
\text { Find } \mathbf{u}^{h, n+1}, \dot{\mathbf{u}}^{h, n+1}, \ddot{\mathbf{u}}^{h, n+1} \in \mathbf{V}^{h} \text { such that: } \\
\mathbf{u}^{h, n+1}=\mathbf{u}^{h, n}+\tau \dot{\mathbf{u}}^{h, n+\frac{1}{2}}, \\
\dot{\mathbf{u}}^{h, n+1}=\dot{\mathbf{u}}^{h, n}+\tau \ddot{\mathbf{u}}^{h, n+\frac{1}{2}}, \\
\left\langle\rho \ddot{\mathbf{u}}^{h, n+\frac{1}{2}}, \mathbf{v}^{h}\right\rangle+A_{\Theta \gamma_{h}}\left(\mathbf{u}^{h, n+\frac{1}{2}}, \mathbf{v}^{h}\right)+\int_{\Gamma_{C}} \frac{1}{\gamma_{h}} \Phi\left(\mathbf{u}^{h, n}, \mathbf{u}^{h, n+1}\right) P_{\Theta \gamma_{h}}\left(\mathbf{v}^{h}\right) \mathrm{d} \Gamma=L^{n+\frac{1}{2}}\left(\mathbf{v}^{h}\right), \\
\quad \forall \mathbf{v}^{h} \in \mathbf{V}^{h},
\end{array}\right.
$$


with the initial conditions $\mathbf{u}^{h, 0}=\mathbf{u}_{0}^{h}, \dot{\mathbf{u}}^{h, 0}=\dot{\mathbf{u}}_{0}^{h}, \ddot{\mathbf{u}}^{h, 0}=\ddot{\mathbf{u}}_{0}^{h}$ (see Rem. 2.1) and with the following expression for $\Phi\left(\mathbf{u}^{h, n}, \mathbf{u}^{h, n+1}\right)$ :

$$
\Phi\left(\mathbf{u}^{h, n}, \mathbf{u}^{h, n+1}\right):=H\left(P_{\gamma_{h}}\left(\mathbf{u}^{h, n}\right)\right)\left[P_{\gamma_{h}}\left(\mathbf{u}^{h, n+\frac{1}{2}}\right)\right]_{+}+H\left(-P_{\gamma_{h}}\left(\mathbf{u}^{h, n}\right)\right)\left[P_{\gamma_{h}}\left(\mathbf{u}^{h}\right)\right]_{+}^{n+\frac{1}{2}} .
$$

We observe that $\left[P_{\gamma_{h}}\left(\mathbf{u}^{h}\right)\right]_{+}^{n+\frac{1}{2}}=\frac{1}{2}\left(\left[P_{\gamma_{h}}\left(\mathbf{u}^{h, n}\right)\right]_{+}+\left[P_{\gamma_{h}}\left(\mathbf{u}^{h, n+1}\right)\right]_{+}\right)$represents the Crank-Nicolson part, whereas $\left[P_{\gamma_{h}}\left(\mathbf{u}^{h, n+\frac{1}{2}}\right)\right]_{+}=\left[\frac{1}{2}\left(P_{\gamma_{h}}\left(\mathbf{u}^{h, n}\right)+P_{\gamma_{h}}\left(\mathbf{u}^{h, n+1}\right)\right)\right]_{+}$stands for the Midpoint part. So, when $P_{\gamma_{h}}\left(\mathbf{u}^{h, n}\right)>0$, the Midpoint scheme is applied, and when $P_{\gamma_{h}}\left(\mathbf{u}^{h, n}\right)<0$, the Crank-Nicolson scheme is applied instead. When $P_{\gamma_{h}}\left(\mathbf{u}^{h, n}\right)=0$ both schemes coincide.

We recall (see the proof in [5]) that the schemes (2.6) (resp. (2.7) and (2.8)) are well-posed when $(1+\Theta)^{2} \gamma_{0}<$ $C\left(1+\left(\rho h^{2}\right) /\left(\tau^{2} \theta^{2}\right)\right)\left(\right.$ resp. $(1+\Theta)^{2} \gamma_{0}<C\left(1+\left(\rho h^{2}\right) /\left(\tau^{2} \beta\right)\right)$ and $(1+\Theta)^{2} \gamma_{0}<C\left(1+\left(\rho h^{2}\right) / \tau^{2}\right)$.

\section{Energy estimates AND stability}

In this section we will define the following energies:

$$
E^{h, n}:=\frac{1}{2} \rho\left\|\dot{\mathbf{u}}^{h, n}\right\|_{0, \Omega}^{2}+\frac{1}{2} a\left(\mathbf{u}^{h, n}, \mathbf{u}^{h, n}\right),
$$

which is associated with the solution $\mathbf{u}^{h, n}$ of problems (2.6), (2.7) or (2.8). Set also

$$
E_{\Theta}^{h, n}:=E^{h, n}-\frac{\Theta}{2}\left[\left\|\gamma_{h}^{\frac{1}{2}} \sigma_{n}\left(\mathbf{u}^{h, n}\right)\right\|_{0, \Gamma_{C}}^{2}-\left\|\gamma_{h}{ }^{-\frac{1}{2}}\left[P_{\gamma_{h}}\left(\mathbf{u}^{h, n}\right)\right]_{+}\right\|_{0, \Gamma_{C}}^{2}\right]:=E^{h, n}-\Theta R^{h, n} .
$$

Note that the energies $E^{h, n}$ and $E_{\Theta}^{h, n}$ are the fully discrete counterparts of the semi-discrete energies $E^{h}(t)$ and $E_{\Theta}^{h}(t)$ studied in [5].

In order to slightly simplify the notations in the energy estimates below, we will make use of the convention: $P^{n}:=P_{\gamma_{h}}\left(\mathbf{u}^{h, n}\right)$ for any $n \in \mathbb{N}$.

\subsection{Energy estimates for the $\theta$-scheme}

We start with the following proposition for problem (2.6) where the $\theta$-scheme is combined with Nitsche's method.

Proposition 3.1. Suppose that $L^{n} \equiv 0$ for all $n \geq 0$ and that problem (2.6) is well-posed. The following energy identity holds for all $n \geq 0$ :

$$
\begin{aligned}
E_{\Theta}^{h, n+1}-E_{\Theta}^{h, n}= & \left(\frac{1}{2}-\theta\right)\left[\rho\left\|\dot{\mathbf{u}}^{h, n+1}-\dot{\mathbf{u}}^{h, n}\right\|_{0, \Omega}^{2}+A_{\Theta \gamma_{h}}\left(\mathbf{u}^{h, n+1}-\mathbf{u}^{h, n}, \mathbf{u}^{h, n+1}-\mathbf{u}^{h, n}\right)\right. \\
& \left.+\Theta\left\|\gamma_{h}{ }^{-\frac{1}{2}}\left(\left[P^{n+1}\right]_{+}-\left[P^{n}\right]_{+}\right)\right\|_{0, \Gamma_{C}}^{2}\right] \\
& +\Theta \int_{\Gamma_{C}} \frac{1}{\gamma_{h}}\left((1-\theta)\left[P^{n}\right]_{+}\left[P^{n+1}\right]_{-}-\theta\left[P^{n+1}\right]_{+}\left[P^{n}\right]_{-}\right) \mathrm{d} \Gamma \\
& +(\Theta-1) \int_{\Gamma_{C}} \frac{1}{\gamma_{h}}\left(\theta\left[P^{n+1}\right]_{+}+(1-\theta)\left[P^{n}\right]_{+}\right)\left(u_{n}^{h, n+1}-u_{n}^{h, n}\right) \mathrm{d} \Gamma .
\end{aligned}
$$

Proof. Using the definition of $E_{\Theta}^{h, n+1}, E_{\Theta}^{h, n}$ and $A_{\Theta \gamma_{h}}$, we get

$$
\begin{aligned}
E_{\Theta}^{h, n+1}-E_{\Theta}^{h, n}= & \frac{\rho}{2}\left\langle\dot{\mathbf{u}}^{h, n+1}+\dot{\mathbf{u}}^{h, n}, \dot{\mathbf{u}}^{h, n+1}-\dot{\mathbf{u}}^{h, n}\right\rangle+\frac{1}{2} A_{\Theta \gamma_{h}}\left(\mathbf{u}^{h, n+1}+\mathbf{u}^{h, n}, \mathbf{u}^{h, n+1}-\mathbf{u}^{h, n}\right) \\
& +\frac{\Theta}{2} \int_{\Gamma_{C}} \frac{1}{\gamma_{h}}\left(\left[P^{n+1}\right]_{+}^{2}-\left[P^{n}\right]_{+}^{2}\right) \mathrm{d} \Gamma .
\end{aligned}
$$


From the definition of the scheme in (2.6), we have $\mathbf{u}^{h, n+1}-\mathbf{u}^{h, n}=\tau \dot{\mathbf{u}}^{h, n+\theta}$. So

$$
\frac{2}{\tau}\left(\mathbf{u}^{h, n+1}-\mathbf{u}^{h, n}\right)=2\left(\theta \dot{\mathbf{u}}^{h, n+1}+(1-\theta) \dot{\mathbf{u}}^{h, n}\right)=\left(\dot{\mathbf{u}}^{h, n+1}+\dot{\mathbf{u}}^{h, n}\right)+(2 \theta-1)\left(\dot{\mathbf{u}}^{h, n+1}-\dot{\mathbf{u}}^{h, n}\right)
$$

which yields the identity

$$
\dot{\mathbf{u}}^{h, n+1}+\dot{\mathbf{u}}^{h, n}=\frac{2}{\tau}\left(\mathbf{u}^{h, n+1}-\mathbf{u}^{h, n}\right)+(1-2 \theta)\left(\dot{\mathbf{u}}^{h, n+1}-\dot{\mathbf{u}}^{h, n}\right) .
$$

The first term in (3.2) is handled by using (3.3):

$\frac{\rho}{2}\left\langle\dot{\mathbf{u}}^{h, n+1}+\dot{\mathbf{u}}^{h, n}, \dot{\mathbf{u}}^{h, n+1}-\dot{\mathbf{u}}^{h, n}\right\rangle=\rho\left(\frac{1}{2}-\theta\right)\left\|\dot{\mathbf{u}}^{h, n+1}-\dot{\mathbf{u}}^{h, n}\right\|_{0, \Omega}^{2}+\frac{\rho}{\tau}\left\langle\mathbf{u}^{h, n+1}-\mathbf{u}^{h, n}, \dot{\mathbf{u}}^{h, n+1}-\dot{\mathbf{u}}^{h, n}\right\rangle$.

Besides, using again the definition of the scheme in (2.6), we have for any $\mathbf{v} \in \mathbf{V}^{h}$ :

$$
\frac{\rho}{\tau}\left\langle\dot{\mathbf{u}}^{h, n+1}-\dot{\mathbf{u}}^{h, n}, \mathbf{v}\right\rangle=\rho\left\langle\ddot{\mathbf{u}}^{h, n+\theta}, \mathbf{v}\right\rangle=\rho \theta\left\langle\ddot{\mathbf{u}}^{h, n+1}, \mathbf{v}\right\rangle+\rho(1-\theta)\left\langle\ddot{\mathbf{u}}^{h, n}, \mathbf{v}\right\rangle .
$$

By using again the definition of the scheme, expression (3.5) becomes :

$$
\begin{aligned}
\frac{\rho}{\tau}\left\langle\dot{\mathbf{u}}^{h, n+1}-\dot{\mathbf{u}}^{h, n}, \mathbf{v}\right\rangle= & -\theta A_{\Theta \gamma_{h}}\left(\mathbf{u}^{h, n+1}, \mathbf{v}\right)-\theta \int_{\Gamma_{C}} \frac{1}{\gamma_{h}}\left[P^{n+1}\right]_{+} P_{\Theta \gamma_{h}}(\mathbf{v}) \mathrm{d} \Gamma \\
& -(1-\theta) A_{\Theta \gamma_{h}}\left(\mathbf{u}^{h, n}, \mathbf{v}\right)-(1-\theta) \int_{\Gamma_{C}} \frac{1}{\gamma_{h}}\left[P^{n}\right]_{+} P_{\Theta \gamma_{h}}(\mathbf{v}) \mathrm{d} \Gamma .
\end{aligned}
$$

Using expression (3.6) with $\mathbf{v}=\mathbf{u}^{h, n+1}-\mathbf{u}^{h, n}$, putting the result in (3.4) and then in (3.2) gives :

$$
\begin{aligned}
E_{\Theta}^{h, n+1}-E_{\Theta}^{h, n}= & \rho\left(\frac{1}{2}-\theta\right)\left\|\dot{\mathbf{u}}^{h, n+1}-\dot{\mathbf{u}}^{h, n}\right\|_{0, \Omega}^{2}-\theta A_{\Theta \gamma_{h}}\left(\mathbf{u}^{h, n+1}, \mathbf{u}^{h, n+1}-\mathbf{u}^{h, n}\right) \\
& -\theta \int_{\Gamma_{C}} \frac{1}{\gamma_{h}}\left[P^{n+1}\right]_{+} P_{\Theta \gamma_{h}}\left(\mathbf{u}^{h, n+1}-\mathbf{u}^{h, n}\right) \mathrm{d} \Gamma \\
& -(1-\theta) A_{\Theta \gamma_{h}}\left(\mathbf{u}^{h, n}, \mathbf{u}^{h, n+1}-\mathbf{u}^{h, n}\right) \\
& -(1-\theta) \int_{\Gamma_{C}} \frac{1}{\gamma_{h}}\left[P^{n}\right]_{+} P_{\Theta \gamma_{h}}\left(\mathbf{u}^{h, n+1}-\mathbf{u}^{h, n}\right) \mathrm{d} \Gamma \\
& +\frac{1}{2} A_{\Theta \gamma_{h}}\left(\mathbf{u}^{h, n+1}+\mathbf{u}^{h, n}, \mathbf{u}^{h, n+1}-\mathbf{u}^{h, n}\right) \\
& +\frac{\Theta}{2} \int_{\Gamma_{C}} \frac{1}{\gamma_{h}}\left(\left[P^{n+1}\right]_{+}^{2}-\left[P^{n}\right]_{+}^{2}\right) \mathrm{d} \Gamma \\
= & \left(\frac{1}{2}-\theta\right)\left(\rho\left\|\dot{\mathbf{u}}^{h, n+1}-\dot{\mathbf{u}}^{h, n}\right\|_{0, \Omega}^{2}+A_{\Theta \gamma_{h}}\left(\mathbf{u}^{h, n+1}-\mathbf{u}^{h, n}, \mathbf{u}^{h, n+1}-\mathbf{u}^{h, n}\right)\right) \\
& -\int_{\Gamma_{C}} \frac{1}{\gamma_{h}}\left(\theta\left[P^{n+1}\right]_{+}+(1-\theta)\left[P^{n}\right]_{+}\right) P_{\Theta \gamma_{h}}\left(\mathbf{u}^{h, n+1}-\mathbf{u}^{h, n}\right) \mathrm{d} \Gamma \\
& +\frac{\Theta}{2} \int_{\Gamma_{C}} \frac{1}{\gamma_{h}}\left(\left[P^{n+1}\right]_{+}^{2}-\left[P^{n}\right]_{+}^{2}\right) \mathrm{d} \Gamma \\
&
\end{aligned}
$$


It remains then to estimate the last two integral terms in (3.7). Writing

$$
P_{\Theta \gamma_{h}}\left(\mathbf{u}^{h, n+1}-\mathbf{u}^{h, n}\right)=\Theta P_{\gamma_{h}}\left(\mathbf{u}^{h, n+1}-\mathbf{u}^{h, n}\right)+(1-\Theta)\left(u_{n}^{h, n+1}-u_{n}^{h, n}\right),
$$

and using the identities (2.2) and (2.3) we obtain:

$$
\begin{aligned}
& -\int_{\Gamma_{C}} \frac{1}{\gamma_{h}}\left(\theta\left[P^{n+1}\right]_{+}+(1-\theta)\left[P^{n}\right]_{+}\right) P_{\Theta \gamma_{h}}\left(\mathbf{u}^{h, n+1}-\mathbf{u}^{h, n}\right) \mathrm{d} \Gamma \\
& +\frac{\Theta}{2} \int_{\Gamma_{C}} \frac{1}{\gamma_{h}}\left(\left[P^{n+1}\right]_{+}^{2}-\left[P^{n}\right]_{+}^{2}\right) \mathrm{d} \Gamma \\
= & \left(\frac{1}{2}-\theta\right) \Theta\left\|\gamma_{h}^{-\frac{1}{2}}\left(\left[P^{n+1}\right]_{+}-\left[P^{n}\right]_{+}\right)\right\|_{0, \Gamma_{C}}^{2} \\
& +\Theta \int_{\Gamma_{C}} \frac{1}{\gamma_{h}}\left((1-\theta)\left[P^{n}\right]_{+}\left[P^{n+1}\right]_{-}-\theta\left[P^{n+1}\right]_{+}\left[P^{n}\right]_{-}\right) \mathrm{d} \Gamma \\
& +(\Theta-1) \int_{\Gamma_{C}} \frac{1}{\gamma_{h}}\left(\theta\left[P^{n+1}\right]_{+}+(1-\theta)\left[P^{n}\right]_{+}\right)\left(u_{n}^{h, n+1}-u_{n}^{h, n}\right) \mathrm{d} \Gamma .
\end{aligned}
$$

That concludes the proof of (3.1).

A straightforward byproduct of the above result is that the backward Euler scheme $(\theta=1)$ preserves the energy $E_{1}^{h, n}$ for the symmetric Nitsche's method $(\Theta=1)$, which is stated below:

Corollary 3.2. Suppose that $L^{n} \equiv 0$ for all $n \geq 0$. Then, for $\gamma_{0}$ sufficiently small, $\Theta=1$ and $\theta=1$ (backward Euler scheme), the following stability estimate holds for the solution of problem (2.6), for all $n \geq 0$ :

$$
\begin{aligned}
E_{1}^{h, n+1}-E_{1}^{h, n}= & -\frac{1}{2} \rho\left\|\dot{\mathbf{u}}^{h, n+1}-\dot{\mathbf{u}}^{h, n}\right\|_{0, \Omega}^{2}-\frac{1}{2} A_{\gamma_{h}}\left(\mathbf{u}^{h, n+1}-\mathbf{u}^{h, n}, \mathbf{u}^{h, n+1}-\mathbf{u}^{h, n}\right) \\
& -\frac{1}{2}\left\|\gamma_{h}{ }^{-\frac{1}{2}}\left(\left[P^{n+1}\right]_{+}-\left[P^{n}\right]_{+}\right)\right\|_{0, \Gamma_{C}}^{2}-\int_{\Gamma_{C}} \frac{1}{\gamma_{h}}\left(\left[P^{n+1}\right]_{+}\left[P^{n}\right]_{-}\right) \mathrm{d} \Gamma \\
\leq & 0 .
\end{aligned}
$$

Consequently, the scheme (2.6) is unconditionally stable when $\Theta=1, \theta=1$ (i.e., stable for all $h>0$ and all $\tau>0)$.

Proof. Set $\Theta=\theta=1$ in $(3.1)$ and note that $A_{\gamma_{h}}(\cdot, \cdot)$ is an inner product in $\mathbf{V}^{h}$ whenever $\gamma_{0}$ is small enough.

Remark 3.3. In contrast with the backward Euler case, in the Crank-Nicolson case $\left(\theta=\frac{1}{2}\right)$, and for the symmetric version $(\Theta=1)$, a positive term in $\left[P^{n}\right]_{+}\left[P^{n+1}\right]_{-}$remains in the right part in the estimate (3.1), so we cannot conclude to unconditional stability. In Section 4, numerical experiments will show that energy can be created and so the scheme can be indeed unstable for impact problems. When $\Theta \neq 1$, we did not manage yet to obtain stability results. 


\subsection{Energy estimates for the Newmark scheme}

With similar arguments as in [18] we establish also an energy estimate for problem (2.7) where the Newmark scheme is combined with Nitsche's method.

Proposition 3.4. Suppose that $L^{n} \equiv 0$ for all $n \geq 0$ and that problem (2.7) is well-posed. The following energy identity holds for all $n \geq 0$ :

$$
\begin{aligned}
E_{\Theta}^{h, n+1}-E_{\Theta}^{h, n}= & \left(\frac{1}{2}-\gamma\right)\left[A_{\Theta \gamma_{h}}\left(\mathbf{u}^{h, n+1}-\mathbf{u}^{h, n}, \mathbf{u}^{h, n+1}-\mathbf{u}^{h, n}\right)\right. \\
& \left.+\Theta\left\|\gamma_{h}-\frac{1}{2}\left(\left[P^{n+1}\right]_{+}-\left[P^{n}\right]_{+}\right)\right\|_{0, \Gamma_{C}}^{2}\right] \\
& +\Theta \int_{\Gamma_{C}} \frac{1}{\gamma_{h}}\left((1-\gamma)\left[P^{n}\right]_{+}\left[P^{n+1}\right]_{-}-\gamma\left[P^{n+1}\right]_{+}\left[P^{n}\right]_{-}\right) \mathrm{d} \Gamma \\
& +\tau\left(\beta-\frac{\gamma}{2}\right)\left[A_{\Theta \gamma_{h}}\left(\mathbf{u}^{h, n+1}-\mathbf{u}^{h, n}, \dot{\mathbf{u}}^{h, n+1}-\dot{\mathbf{u}}^{h, n}\right)\right. \\
& \left.+\int_{\Gamma_{C}} \frac{1}{\gamma_{h}}\left(\left[P^{n+1}\right]_{+}-\left[P^{n}\right]_{+}\right) P_{\Theta \gamma_{h}}\left(\dot{\mathbf{u}}^{h, n+1}-\dot{\mathbf{u}}^{h, n}\right) \mathrm{d} \Gamma\right] \\
& +(\Theta-1) \int_{\Gamma_{C}} \frac{1}{\gamma_{h}}\left(\gamma\left[P^{n+1}\right]_{+}+(1-\gamma)\left[P^{n}\right]_{+}\right)\left(u_{n}^{h, n+1}-u_{n}^{h, n}\right) \mathrm{d} \Gamma .
\end{aligned}
$$

Proof. Using the definition of $E_{\Theta}^{h, n+1}, E_{\Theta}^{h, n}$ and $A_{\Theta \gamma_{h}}$, we get:

$$
\begin{aligned}
E_{\Theta}^{h, n+1}-E_{\Theta}^{h, n}= & \frac{\rho}{2}\left\langle\dot{\mathbf{u}}^{h, n+1}-\dot{\mathbf{u}}^{h, n}, \dot{\mathbf{u}}^{h, n}+\dot{\mathbf{u}}^{h, n+1}\right\rangle+\frac{1}{2} A_{\Theta \gamma_{h}}\left(\mathbf{u}^{h, n+1}-\mathbf{u}^{h, n}, \mathbf{u}^{h, n}+\mathbf{u}^{h, n+1}\right) \\
& +\frac{\Theta}{2} \int_{\Gamma_{C}} \frac{1}{\gamma_{h}}\left(\left[P^{n+1}\right]_{+}^{2}-\left[P^{n}\right]_{+}^{2}\right) \mathrm{d} \Gamma .
\end{aligned}
$$

With the first equation of (2.7), and then the third equation of (2.7) we obtain for $\mathbf{v}^{h} \in \mathbf{V}^{h}$ :

$$
\begin{aligned}
\rho\left\langle-\mathbf{u}^{h, n+1}+\mathbf{u}^{h, n}+\tau \dot{\mathbf{u}}^{h, n}, \mathbf{v}^{h}\right\rangle= & -\rho \frac{\tau^{2}}{2}\left((1-2 \beta)\left\langle\ddot{\mathbf{u}}^{h, n}, \mathbf{v}^{h}\right\rangle+2 \beta\left\langle\ddot{\mathbf{u}}^{h, n+1}, \mathbf{v}^{h}\right\rangle\right) \\
= & \tau^{2}\left(\frac{1}{2}-\beta\right)\left(A_{\Theta \gamma_{h}}\left(\mathbf{u}^{h, n}, \mathbf{v}^{h}\right)+\int_{\Gamma_{C}} \frac{1}{\gamma_{h}}\left[P^{n}\right]_{+} P_{\Theta \gamma_{h}}\left(\mathbf{v}^{h}\right) \mathrm{d} \Gamma\right) \\
& +\tau^{2} \beta\left(A_{\Theta \gamma_{h}}\left(\mathbf{u}^{h, n+1}, \mathbf{v}^{h}\right)+\int_{\Gamma_{C}} \frac{1}{\gamma_{h}}\left[P^{n+1}\right]_{+} P_{\Theta \gamma_{h}}\left(\mathbf{v}^{h}\right) \mathrm{d} \Gamma\right) .
\end{aligned}
$$

Similarly with the second equation and then the third equation of (2.7) we get for $\mathbf{v}^{h} \in \mathbf{V}^{h}$ :

$$
\begin{aligned}
\rho\left\langle\dot{\mathbf{u}}^{h, n+1}-\dot{\mathbf{u}}^{h, n}, \mathbf{v}^{h}\right\rangle= & \rho \tau\left((1-\gamma)\left\langle\ddot{\mathbf{u}}^{h, n}, \mathbf{v}^{h}\right\rangle+\gamma\left\langle\ddot{\mathbf{u}}^{h, n+1}, \mathbf{v}^{h}\right\rangle\right) \\
= & \tau(\gamma-1)\left(A_{\Theta \gamma_{h}}\left(\mathbf{u}^{h, n}, \mathbf{v}^{h}\right)+\int_{\Gamma_{C}} \frac{1}{\gamma_{h}}\left[P^{n}\right]_{+} P_{\Theta \gamma_{h}}\left(\mathbf{v}^{h}\right) \mathrm{d} \Gamma\right) \\
& -\tau \gamma\left(A_{\Theta \gamma_{h}}\left(\mathbf{u}^{h, n+1}, \mathbf{v}^{h}\right)+\int_{\Gamma_{C}} \frac{1}{\gamma_{h}}\left[P^{n+1}\right]_{+} P_{\Theta \gamma_{h}}\left(\mathbf{v}^{h}\right) \mathrm{d} \Gamma\right) .
\end{aligned}
$$

We sum (3.12) with $\frac{\tau}{2}$ times equation (3.13) which yields:

$$
\begin{aligned}
\rho\left\langle\mathbf{u}^{h, n}-\mathbf{u}^{h, n+1}+\frac{\tau}{2}\left(\dot{\mathbf{u}}^{h, n}+\dot{\mathbf{u}}^{h, n+1}\right), \mathbf{v}^{h}\right\rangle= & \tau^{2}\left(\frac{\gamma}{2}-\beta\right)\left(A_{\Theta \gamma_{h}}\left(\mathbf{u}^{h, n}, \mathbf{v}^{h}\right)+\int_{\Gamma_{C}} \frac{1}{\gamma_{h}}\left[P^{n}\right]_{+} P_{\Theta \gamma_{h}}\left(\mathbf{v}^{h}\right) \mathrm{d} \Gamma\right) \\
& +\tau^{2}\left(\beta-\frac{\gamma}{2}\right)\left(A_{\Theta \gamma_{h}}\left(\mathbf{u}^{h, n+1}, \mathbf{v}^{h}\right)+\int_{\Gamma_{C}} \frac{1}{\gamma_{h}}\left[P^{n+1}\right]_{+} P_{\Theta \gamma_{h}}\left(\mathbf{v}^{h}\right) \mathrm{d} \Gamma\right)
\end{aligned}
$$


Let us reorder the above expression:

$$
\begin{aligned}
\rho\left\langle\dot{\mathbf{u}}^{h, n}+\dot{\mathbf{u}}^{h, n+1}, \mathbf{v}^{h}\right\rangle= & \frac{2 \rho}{\tau}\left\langle\mathbf{u}^{h, n+1}-\mathbf{u}^{h, n}, \mathbf{v}^{h}\right\rangle+2 \tau\left(\beta-\frac{\gamma}{2}\right)\left[A_{\Theta \gamma_{h}}\left(\mathbf{u}^{h, n+1}-\mathbf{u}^{h, n}, \mathbf{v}^{h}\right)\right. \\
& \left.+\int_{\Gamma_{C}} \frac{1}{\gamma_{h}}\left(\left[P^{n+1}\right]_{+}-\left[P^{n}\right]_{+}\right) P_{\Theta \gamma_{h}}\left(\mathbf{v}^{h}\right) \mathrm{d} \Gamma\right] .
\end{aligned}
$$

Taking $\mathbf{v}^{h}=\dot{\mathbf{u}}^{h, n+1}-\dot{\mathbf{u}}^{h, n}$ and using (3.14) into (3.11) yields:

$$
\begin{aligned}
E_{\Theta}^{h, n+1}-E_{\Theta}^{h, n}= & \frac{\rho}{\tau}\left\langle\mathbf{u}^{h, n+1}-\mathbf{u}^{h, n}, \dot{\mathbf{u}}^{h, n+1}-\dot{\mathbf{u}}^{h, n}\right\rangle+\tau\left(\beta-\frac{\gamma}{2}\right)\left[A_{\Theta \gamma_{h}}\left(\mathbf{u}^{h, n+1}-\mathbf{u}^{h, n}, \dot{\mathbf{u}}^{h, n+1}-\dot{\mathbf{u}}^{h, n}\right)\right. \\
& \left.+\int_{\Gamma_{C}} \frac{1}{\gamma_{h}}\left(\left[P^{n+1}\right]_{+}-\left[P^{n}\right]_{+}\right) P_{\Theta \gamma_{h}}\left(\dot{\mathbf{u}}^{h, n+1}-\dot{\mathbf{u}}^{h, n}\right) \mathrm{d} \Gamma\right] \\
& +\frac{1}{2} A_{\Theta \gamma_{h}}\left(\mathbf{u}^{h, n+1}-\mathbf{u}^{h, n}, \mathbf{u}^{h, n}+\mathbf{u}^{h, n+1}\right)+\frac{\Theta}{2} \int_{\Gamma_{C}} \frac{1}{\gamma_{h}}\left(\left[P^{n+1}\right]_{+}^{2}-\left[P^{n}\right]_{+}^{2}\right) \mathrm{d} \Gamma .
\end{aligned}
$$

We rewrite the first term in the above expression using (3.13), with $\mathbf{v}^{h}=\mathbf{u}^{h, n+1}-\mathbf{u}^{h, n}$ :

$$
\begin{aligned}
E_{\Theta}^{h, n+1}-E_{\Theta}^{h, n}= & (\gamma-1)\left(A_{\Theta \gamma_{h}}\left(\mathbf{u}^{h, n}, \mathbf{u}^{h, n+1}-\mathbf{u}^{h, n}\right)\right. \\
& \left.+\int_{\Gamma_{C}} \frac{1}{\gamma_{h}}\left[P^{n}\right]_{+} P_{\Theta \gamma_{h}}\left(\mathbf{u}^{h, n+1}-\mathbf{u}^{h, n}\right) \mathrm{d} \Gamma\right) \\
& -\gamma\left(A_{\Theta \gamma_{h}}\left(\mathbf{u}^{h, n+1}, \mathbf{u}^{h, n+1}-\mathbf{u}^{h, n}\right)+\int_{\Gamma_{C}} \frac{1}{\gamma_{h}}\left[P^{n+1}\right]_{+} P_{\Theta \gamma_{h}}\left(\mathbf{u}^{h, n+1}-\mathbf{u}^{h, n}\right) \mathrm{d} \Gamma\right) \\
& +\tau\left(\beta-\frac{\gamma}{2}\right)\left[A_{\Theta \gamma_{h}}\left(\mathbf{u}^{h, n+1}-\mathbf{u}^{h, n}, \dot{\mathbf{u}}^{h, n+1}-\dot{\mathbf{u}}^{h, n}\right)\right. \\
& \left.+\int_{\Gamma_{C}} \frac{1}{\gamma_{h}}\left(\left[P^{n+1}\right]_{+}-\left[P^{n}\right]_{+}\right) P_{\Theta \gamma_{h}}\left(\dot{\mathbf{u}}^{h, n+1}-\dot{\mathbf{u}}^{h, n}\right) \mathrm{d} \Gamma\right] \\
& +\frac{1}{2} A_{\Theta \gamma_{h}}\left(\mathbf{u}^{h, n+1}-\mathbf{u}^{h, n}, \mathbf{u}^{h, n}+\mathbf{u}^{h, n+1}\right)+\frac{\Theta}{2} \int_{\Gamma_{C}} \frac{1}{\gamma_{h}}\left(\left[P^{n+1}\right]_{+}^{2}-\left[P^{n}\right]_{+}^{2}\right) \mathrm{d} \Gamma .
\end{aligned}
$$

We reorder all the terms and use property (3.8):

$$
\begin{aligned}
E_{\Theta}^{h, n+1}-E_{\Theta}^{h, n}= & \left(\frac{1}{2}-\gamma\right) A_{\Theta \gamma_{h}}\left(\mathbf{u}^{h, n+1}-\mathbf{u}^{h, n}, \mathbf{u}^{h, n+1}-\mathbf{u}^{h, n}\right) \\
& +(\gamma-1) \Theta \int_{\Gamma_{C}} \frac{1}{\gamma_{h}}\left[P^{n}\right]_{+}\left(P^{n+1}-P^{n}\right) \mathrm{d} \Gamma \\
& -\gamma \Theta \int_{\Gamma_{C}} \frac{1}{\gamma_{h}}\left[P^{n+1}\right]_{+}\left(P^{n+1}-P^{n}\right) \mathrm{d} \Gamma \\
& +\frac{\Theta}{2} \int_{\Gamma_{C}} \frac{1}{\gamma_{h}}\left(\left[P^{n+1}\right]_{+}^{2}-\left[P^{n}\right]_{+}^{2}\right) \mathrm{d} \Gamma \\
& +\tau\left(\beta-\frac{\gamma}{2}\right)\left[A_{\Theta \gamma_{h}}\left(\mathbf{u}^{h, n+1}-\mathbf{u}^{h, n}, \dot{\mathbf{u}}^{h, n+1}-\dot{\mathbf{u}}^{h, n}\right)\right. \\
& \left.+\int_{\Gamma_{C}} \frac{1}{\gamma_{h}}\left(\left[P^{n+1}\right]_{+}-\left[P^{n}\right]_{+}\right) P_{\Theta \gamma_{h}}\left(\dot{\mathbf{u}}^{h, n+1}-\dot{\mathbf{u}}^{h, n}\right) \mathrm{d} \Gamma\right] \\
& +(\gamma-1)(1-\Theta) \int_{\Gamma_{C}} \frac{1}{\gamma_{h}}\left[P^{n}\right]_{+}\left(u^{h, n+1}-u^{h, n}\right) \mathrm{d} \Gamma \\
& -\gamma(1-\Theta) \int_{\Gamma_{C}} \frac{1}{\gamma_{h}}\left[P^{n+1}\right]_{+}\left(u^{h, n+1}-u^{h, n}\right) \mathrm{d} \Gamma
\end{aligned}
$$


Using (2.2) and (2.3), this expression can be simplified into:

$$
\begin{aligned}
E_{\Theta}^{h, n+1}-E_{\Theta}^{h, n}= & \left(\frac{1}{2}-\gamma\right)\left[A_{\Theta \gamma_{h}}\left(\mathbf{u}^{h, n+1}-\mathbf{u}^{h, n}, \mathbf{u}^{h, n+1}-\mathbf{u}^{h, n}\right)+\Theta\left\|\gamma_{h}{ }^{-\frac{1}{2}}\left(\left[P^{n+1}\right]_{+}-\left[P^{n}\right]_{+}\right)\right\|_{0, \Gamma_{C}}^{2}\right] \\
& +\Theta \int_{\Gamma_{C}} \frac{1}{\gamma_{h}}\left((1-\gamma)\left[P^{n}\right]_{+}\left[P^{n+1}\right]_{-}-\gamma\left[P^{n+1}\right]_{+}\left[P^{n}\right]_{-}\right) \mathrm{d} \Gamma \\
& +\tau\left(\beta-\frac{\gamma}{2}\right)\left[A_{\Theta \gamma_{h}}\left(\mathbf{u}^{h, n+1}-\mathbf{u}^{h, n}, \dot{\mathbf{u}}^{h, n+1}-\dot{\mathbf{u}}^{h, n}\right)\right. \\
& \left.+\int_{\Gamma_{C}} \frac{1}{\gamma_{h}}\left(\left[P^{n+1}\right]_{+}-\left[P^{n}\right]_{+}\right) P_{\Theta \gamma_{h}}\left(\dot{\mathbf{u}}^{h, n+1}-\dot{\mathbf{u}}^{h, n}\right) \mathrm{d} \Gamma\right] \\
& +(\Theta-1) \int_{\Gamma_{C}} \frac{1}{\gamma_{h}}\left(\gamma\left[P^{n+1}\right]_{+}+(1-\gamma)\left[P^{n}\right]_{+}\right)\left(u_{n}^{h, n+1}-u_{n}^{h, n}\right) \mathrm{d} \Gamma .
\end{aligned}
$$

We conclude from here that identity (3.10) holds.

A consequence of the above proposition is that Newmark for $\gamma=1$ and $\beta=\frac{1}{2}$ preserves the energy $E_{\Theta}^{h, n}$ for the symmetric Nitsche method $(\Theta=1)$, which is stated below:

Corollary 3.5. Suppose that $L^{n} \equiv 0$ for all $n \geq 0$. Then, for $\gamma_{0}$ sufficiently small, $\Theta=1, \gamma=1$, and $\beta=1 / 2$, the following stability estimate holds for the solution of problem (2.7) for all $n \geq 0$ :

$$
\begin{aligned}
E_{1}^{h, n+1}-E_{1}^{h, n}= & -\frac{1}{2} A_{\Theta \gamma_{h}}\left(\mathbf{u}^{h, n+1}-\mathbf{u}^{h, n}, \mathbf{u}^{h, n+1}-\mathbf{u}^{h, n}\right) \\
& -\frac{1}{2}\left\|\gamma_{h}{ }^{-\frac{1}{2}}\left(\left[P^{n+1}\right]_{+}-\left[P^{n}\right]_{+}\right)\right\|_{0, \Gamma_{C}}^{2}-\int_{\Gamma_{C}} \frac{1}{\gamma_{h}}\left[P^{n+1}\right]_{+}\left[P^{n}\right]_{-} \mathrm{d} \Gamma \\
\leq & 0 .
\end{aligned}
$$

So the scheme (2.7) is unconditionally stable when $\Theta=1, \gamma=1$ and $\beta=\frac{1}{2}$ (i.e., stable for all $h>0$ and all $\tau>0)$.

Proof. The proof of (3.15) is straightforward by using (3.10).

Remark 3.6. The same observations as in Remark 3.3 apply also here, setting $\gamma=\frac{1}{2}$ and $\beta=\frac{1}{4}$ to recover Crank-Nicolson.

\subsection{Energy estimates for the Hybrid scheme}

Proposition 3.7. Suppose that $L^{n} \equiv 0$ for all $n \geq 0$ and that problem (2.8) is well-posed. The following energy identity holds for all $n \geq 0$ :

$$
\begin{aligned}
E_{\Theta}^{h, n+1}-E_{\Theta}^{h, n}= & -\Theta \int_{\Gamma_{C}} \frac{1}{2 \gamma_{h}}\left(H\left(P^{n}\right) H\left(P^{n}+P^{n+1}\right)\left[P^{n+1}\right]_{-}^{2}\right. \\
& \left.+H\left(P^{n}\right) H\left(-P^{n}-P^{n+1}\right)\left[P^{n}\right]_{+}^{2}+\left[P^{n}\right]_{-}\left[P^{n+1}\right]_{+}\right) \mathrm{d} \Gamma \\
& +(\Theta-1) \int_{\Gamma_{C}} \frac{1}{2 \gamma_{h}}\left(H\left(P^{n}\right)\left[P^{n}+P^{n+1}\right]_{+}\right. \\
& \left.+H\left(-P^{n}\right)\left(\left[P^{n}\right]_{+}+\left[P^{n+1}\right]_{+}\right)\right)\left(u_{n}^{h, n+1}-u_{n}^{h, n}\right) \mathrm{d} \Gamma .
\end{aligned}
$$


Proof. From the definitions of $E_{\Theta}^{h, n+1}, E_{\Theta}^{h, n}, A_{\Theta \gamma_{h}}$, the scheme (2.8), and using the test function $\mathbf{v}^{h}=\tau \dot{\mathbf{u}}^{h, n+\frac{1}{2}}$ in Problem (2.8), we get:

$$
\begin{aligned}
E_{\Theta}^{h, n+1}-E_{\Theta}^{h, n}= & \frac{\rho}{2}\left\langle\dot{\mathbf{u}}^{h, n+1}-\dot{\mathbf{u}}^{h, n}, \dot{\mathbf{u}}^{h, n}+\dot{\mathbf{u}}^{h, n+1}\right\rangle \\
& +\frac{1}{2} A_{\Theta \gamma_{h}}\left(\mathbf{u}^{h, n+1}-\mathbf{u}^{h, n}, \mathbf{u}^{h, n}+\mathbf{u}^{h, n+1}\right) \\
& +\frac{\Theta}{2} \int_{\Gamma_{C}} \frac{1}{\gamma_{h}}\left(\left[P^{n+1}\right]_{+}^{2}-\left[P^{n}\right]_{+}^{2}\right) \mathrm{d} \Gamma \\
= & \rho\left\langle\ddot{\mathbf{u}}^{h, n+\frac{1}{2}}, \tau \dot{\mathbf{u}}^{h, n+\frac{1}{2}}\right\rangle \\
& +A_{\Theta \gamma_{h}}\left(\dot{\mathbf{u}}^{h, n+\frac{1}{2}}, \tau \mathbf{u}^{h, n+\frac{1}{2}}\right)+\frac{\Theta}{2} \int_{\Gamma_{C}} \frac{1}{\gamma_{h}}\left(\left[P^{n+1}\right]_{+}^{2}-\left[P^{n}\right]_{+}^{2}\right) \mathrm{d} \Gamma \\
E_{\Theta}^{h, n+1}-E_{\Theta}^{h, n}=- & \int_{\Gamma_{C}} \frac{1}{\gamma_{h}} \Phi\left(\mathbf{u}^{h, n}, \mathbf{u}^{h, n+1}\right) P_{\Theta \gamma_{h}}\left(\tau \dot{\mathbf{u}}^{h, n+\frac{1}{2}}\right) \mathrm{d} \Gamma \\
& +\frac{\Theta}{2} \int_{\Gamma_{C}} \frac{1}{\gamma_{h}}\left(\left[P^{n+1}\right]_{+}^{2}-\left[P^{n}\right]_{+}^{2}\right) \mathrm{d} \Gamma \\
= & -\int_{\Gamma_{C}} \frac{\Theta}{\gamma_{h}} \Phi\left(\mathbf{u}^{h, n}, \mathbf{u}^{h, n+1}\right)\left(P^{n+1}-P^{n}\right) \mathrm{d} \Gamma+\frac{\Theta}{2} \int_{\Gamma_{C}} \frac{1}{\gamma_{h}}\left(\left[P^{n+1}\right]_{+}^{2}-\left[P^{n}\right]_{+}^{2}\right) \mathrm{d} \Gamma \\
& +(\Theta-1) \int_{\Gamma_{C}} \frac{1}{\gamma_{h}} \Phi\left(\mathbf{u}^{h, n}, \mathbf{u}^{h, n+1}\right)\left(u_{n}^{h, n+1}-u_{n}^{h, n}\right) \mathrm{d} \Gamma \\
= & \Theta X+(\Theta-1) Y,
\end{aligned}
$$

with obvious notations for the integrals $X$ and $Y$. Note that the identity (3.8) is used to obtain the last expression. We now consider the function $\Psi$ to be integrated in $X$, namely

$$
\Psi\left(\mathbf{u}^{h, n}, \mathbf{u}^{h, n+1}\right):=-\frac{1}{\gamma_{h}} \Phi\left(\mathbf{u}^{h, n}, \mathbf{u}^{h, n+1}\right)\left(P^{n+1}-P^{n}\right)+\frac{1}{2 \gamma_{h}}\left(\left[P^{n+1}\right]_{+}^{2}-\left[P^{n}\right]_{+}^{2}\right) .
$$

We rewrite $\Psi\left(\mathbf{u}^{h, n}, \mathbf{u}^{h, n+1}\right)$ using the definition of $\Phi\left(\mathbf{u}^{h, n}, \mathbf{u}^{h, n+1}\right)$ in $(2.9)$ :

$$
\begin{aligned}
\Psi\left(\mathbf{u}^{h, n}, \mathbf{u}^{h, n+1}\right)= & -\frac{1}{2 \gamma_{h}}\left(H\left(P^{n}\right)\left[P^{n}+P^{n+1}\right]_{+}+H\left(-P^{n}\right)\left(\left[P^{n}\right]_{+}+\left[P^{n+1}\right]_{+}\right)\right)\left(P^{n+1}-P^{n}\right) \\
& +\frac{1}{2 \gamma_{h}}\left(\left[P^{n+1}\right]_{+}^{2}-\left[P^{n}\right]_{+}^{2}\right) .
\end{aligned}
$$

It is easy to check that expression (3.18) can be formulated in the following equivalent way (it suffices to discuss the five cases $P^{n}<0 ; P^{n}=0 ; P^{n}>0$ and $P^{n}+P^{n+1}>0 ; P^{n}>0$ and $P^{n}+P^{n+1}<0 ; P^{n}>0$ and $\left.P^{n}+P^{n+1}=0\right)$ :

$$
\begin{aligned}
\Psi\left(\mathbf{u}^{h, n}, \mathbf{u}^{h, n+1}\right)= & -\frac{1}{2 \gamma_{h}}\left(H\left(P^{n}\right) H\left(P^{n}+P^{n+1}\right)\left[P^{n+1}\right]_{-}^{2}\right. \\
& \left.+H\left(P^{n}\right) H\left(-P^{n}-P^{n+1}\right)\left[P^{n}\right]_{+}^{2}+\left[P^{n}\right]_{-}\left[P^{n+1}\right]_{+}\right) .
\end{aligned}
$$

Equality (3.19) shows that $\Psi\left(\mathbf{u}^{h, n}, \mathbf{u}^{h, n+1}\right)$ is the sum of three nonpositive terms which implies that $\Psi\left(\mathbf{u}^{h, n}, \mathbf{u}^{h, n+1}\right) \leq 0$ on $\Gamma_{C}$. Estimate (3.16) follows from (3.17), (2.9) and (3.19). 
A consequence of the above proposition is that the Hybrid scheme is dissipative with respect to the energy $E_{\Theta}^{h, n}$ for the symmetric Nitsche method $(\Theta=1)$, which is stated below:

Corollary 3.8. Suppose that $L^{n} \equiv 0$ for all $n \geq 0$ and that problem (2.8) is well-posed. Suppose also that $\Theta=1$. The following stability estimate holds for the solution of problem (2.8) for all $n \geq 0$ :

$$
\begin{aligned}
E_{1}^{h, n+1}-E_{1}^{h, n}= & -\int_{\Gamma_{C}} \frac{1}{2 \gamma_{h}}\left(H\left(P^{n}\right) H\left(P^{n}+P^{n+1}\right)\left[P^{n+1}\right]_{-}^{2}\right. \\
& \left.+H\left(P^{n}\right) H\left(-P^{n}-P^{n+1}\right)\left[P^{n}\right]_{+}^{2}+\left[P^{n}\right]_{-}\left[P^{n+1}\right]_{+}\right) \mathrm{d} \Gamma \\
\leq & 0 .
\end{aligned}
$$

So the scheme (2.8) is unconditionally stable when $\Theta=1$ (i.e., stable for all $h>0$ and all $\tau>0$ ).

Proof. The proof of (3.20) is straightforward by using (3.16) and the nonnegativity of $H$.

Remark 3.9. Note that the energy loss at each time-step for this Hybrid scheme is only due to the contact terms.

\section{NumERICAL EXPERIMENTS}

We first carry out numerical experiments in $1 \mathrm{D}$, where we can compare our results with an analytical solution. Then numerical experiments in $2 \mathrm{D} / 3 \mathrm{D}$ will be described. These numerical tests are performed using the finite element library Getfem $++^{4}$.

Since we study implicit time-marching schemes, a discrete contact problem needs to be solved at each timestep $t^{n}$. To this purpose we make use of a generalized Newton's method, which means that problems (2.6)-(2.8) are derived with respect to $\mathbf{u}^{h}$ to obtain the tangent system. The term generalized Newton's method comes from the fact that the positive part $[x]_{+}$is non-differentiable at $x=0$. However, no special treatment is considered. If a point of non-differentiability is encountered, the tangent system corresponding to one of the two alternatives $(x<0$ or $x>0)$ is chosen arbitrarily. Note that a non-differentiable solution at an integration point is a very rare situation corresponding to what is called a grazing contact (both $u_{n}=0$ and $\sigma_{n}=0$ ). Further details on generalized Newton's method applied to contact problem can be found for instance in [27] and the references therein. For this generalized Newton's method, the parameters will always be: residual-based convergence criterion $\varepsilon^{*}=10^{-8}$ and maximal number of iterations $N_{N}=100$.

\subsection{D numerical experiments: multiple impacts of an elastic bar}

\subsubsection{Setting}

We first deal with the one-dimensional case $d=1$ with a single contact point, namely an elastic bar $\Omega=(0, L)$ with $\Gamma_{C}=\{0\}, \Gamma_{D}=\{L\}$ and $\Gamma_{N}=\emptyset$. The elastodynamic equation is then reduced to find $u: \Omega_{T}=$ $(0, T) \times(0, L) \rightarrow \mathbb{R}$ such that:

$$
\rho \ddot{u}-E \frac{\partial^{2} u}{\partial x^{2}}=f, \quad \text { in } \Omega_{T},
$$

where $E$ is the Young modulus and the Cauchy stress tensor is given by $\sigma(u)=E(\partial u / \partial x)$. Note that $\sigma_{n}(u)=$ $(\sigma(u) n) \cdot n=\sigma(u)$ on $\Gamma_{C}$. In this case, problem (1.1)-(1.2) admits one unique solution (see e.g. [8]) for which the following energy conservation equation holds, for $t$ a.e. in $(0, T)$ :

$$
\frac{1}{2} \frac{\mathrm{d}}{\mathrm{d} t}\left(\int_{\Omega} \rho \dot{u}^{2}(t) \mathrm{d} \Omega+\int_{\Omega} E\left(\frac{\partial u}{\partial x}(t)\right)^{2} \mathrm{~d} \Omega\right)=\int_{\Omega} f(t) \dot{u}(t) \mathrm{d} \Omega .
$$

\footnotetext{
${ }^{4}$ see http://download.gna.org/getfem/html/homepage/
} 
We consider a finite element space (2.1) using linear finite elements $(k=1)$ and a uniform subdivision of $[0, L]$ with $M$ segments (so $L=M h$ ). We denote the vector which contains all the nodal values of $\mathbf{u}^{h, n}$ (resp. $\dot{\mathbf{u}}^{h, n}$ and $\left.\ddot{\mathbf{u}}^{h, n}\right)$ by $\mathbf{U}^{n}:=\left[U_{0}^{n}, \ldots, U_{N}^{n}\right]^{T}$ (resp. $\left.\dot{\mathbf{U}}^{n}, \ddot{\mathbf{U}}^{n}\right)$. The component of index 0 corresponds to the node at the contact point $\Gamma_{C}$. We also note $\mathbf{M}$, resp. $\mathbf{K}$, the mass, resp. the stiffness, matrix that results from the finite element discretization.

We introduce the Courant number which is defined as:

$$
\nu_{C}:=c_{0} \frac{\tau}{h}=\sqrt{\frac{E}{\rho}} \frac{\tau}{h},
$$

where $c_{0}$ is the wave speed associated to the bar. For each simulation, we compute and plot the following time-dependant quantities:

1. The displacement $u$ at the contact point $\Gamma_{C}$, given at time $t^{n}$ by $u^{h, n}(0)\left(=U_{0}^{n}\right)$.

2. The contact pressure $\sigma_{C}$, which, in the discrete case, is different from $\sigma(u)$. If a standard (mixed) method is used for the treatment of contact, it is directly given by the Lagrange multiplier, i.e., $\sigma_{C}^{n}:=\lambda^{h, n}$ at time $t^{n}$. In the case of the Nitsche-based formulation, it can be computed as follows at time $t^{n}$ :

$$
\sigma_{C}^{n}:=-\frac{1}{\gamma_{h}}\left[\left(-u^{h, n}(0)\right)-\gamma_{h} \sigma_{n}\left(u^{h, n}\right)(0)\right]_{+}=-\frac{1}{\gamma_{h}}\left[-U_{0}^{n}-\gamma_{h} \frac{E}{h}\left(U_{1}^{n}-U_{0}^{n}\right)\right]_{+},
$$

which comes from the contact condition $\sigma_{n}(u)=-\frac{1}{\gamma}\left[P_{\gamma}(u)\right]_{+}($see $[5])$.

3. The discrete energy $E^{h}$ at time $t^{n}$

$$
E^{h, n}:=\frac{1}{2}\left(\left(\dot{\mathbf{U}}^{n}\right)^{\mathrm{T}} \mathbf{M} \dot{\mathbf{U}}^{n}+\left(\mathbf{U}^{n}\right)^{\mathrm{T}} \mathbf{K} \mathbf{U}^{n}\right)
$$

and the discrete augmented energy $E_{\Theta}^{h}$ at time $t^{n}$ :

$$
\begin{aligned}
E_{\Theta}^{h, n} & =E^{h, n}-\Theta R^{h, n}, \\
R^{h, n} & =\frac{1}{2} \gamma_{h}\left(\left(\sigma_{n}\left(u^{h, n}\right)(0)\right)^{2}-\left(\sigma_{C}^{n}\right)^{2}\right) .
\end{aligned}
$$

\subsubsection{Numerical results and discussion}

We propose a benchmark associated with multiple impacts which allows us to check both the presence of spurious oscillations and the long term energetic behaviour of the method. In the absence of external volume forces, the bar is initially compressed. It is then released without initial velocity. It first impacts the rigid ground, located at $x=0$, and then gets again compressed.

So we solve equation (4.1) setting $f=0$. We take the following values for the parameters: $E=1, \rho=1$, $L=1, u_{0}(x)=\frac{1}{2}-\frac{x}{2}$ and $\dot{u}_{0}(x)=0$. This problem admits a closed-form solution $u$ which derivation and expression are detailed in [7]. Especially it has a periodic motion of period 3. At each period, the bar remains in contact with the rigid ground during one time unit (see Fig. 1). The chosen simulation time is $T=12$, so that we can observe 4 successive impacts.

We discretize the bar with $M=100$ finite elements $(h=0.01)$ and take $\tau=0.015$. The resulting Courant number is $\nu_{C}=1.5$. 


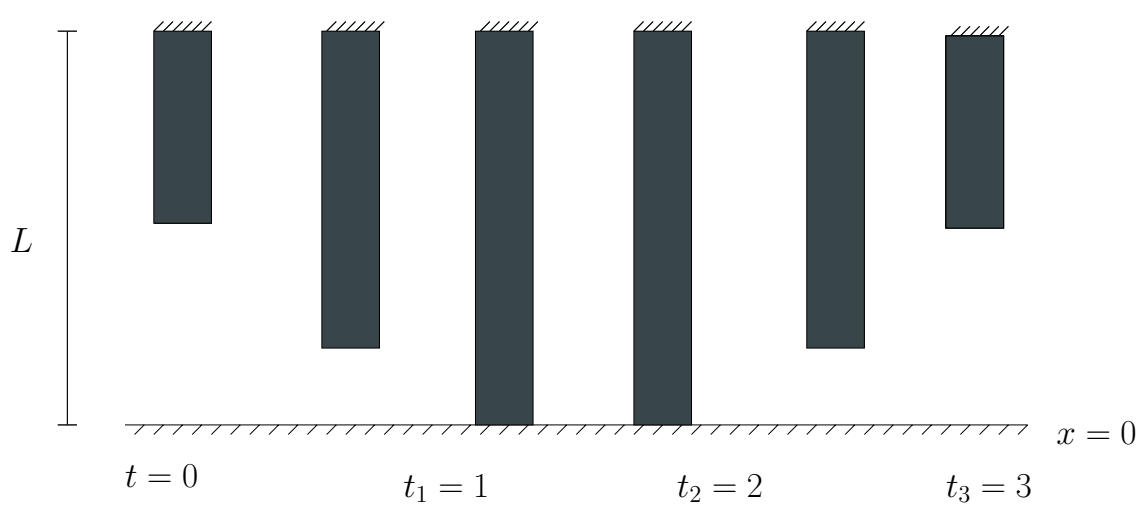

Figure 1. Multiple impacts of an elastic bar. The bar is clamped at $x=L$ and the contact node is located at the bottom. The solution is periodic of period 3, with one impact during each period (here between $t=1$ and $t=2$ ).
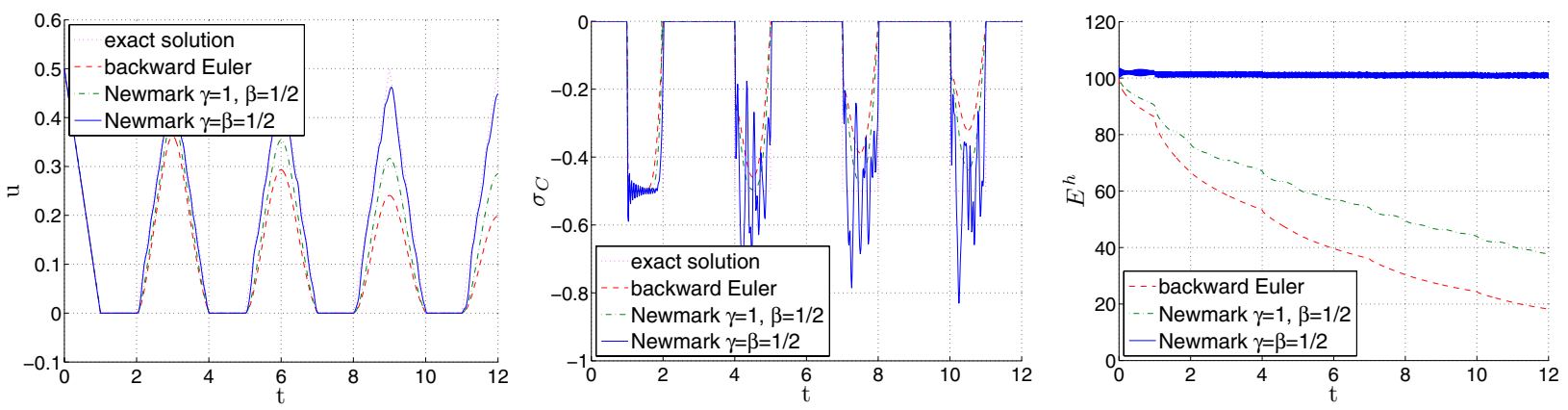

Figure 2. Multiple impacts with Nitsche $(\Theta=1)$. Comparison of backward Euler, Newmark $\left(\gamma=1, \beta=\frac{1}{2}\right)$ and Newmark $\left(\gamma=\beta=\frac{1}{2}\right)$. Displacement $u$ (left), contact pressure $\sigma_{C}$ (center) and discrete energy $E^{h}$ (right).

\subsubsection{Behaviour of diffusive schemes}

We first investigate the most diffusive schemes for which theoretical stability results are available. We recall that these schemes introduce numerical dissipation even in the linear regime (i.e., when contact constraints are not activated). To give an idea, we choose the symmetric variant of the Nitsche method $(\Theta=1)$ with a small parameter $\gamma_{0}=10^{-6}$. We investigate backward Euler $(\theta$-scheme with $\theta=1)$, Newmark with $\gamma=1$ and $\beta=\frac{1}{2}$, and Newmark with $\gamma=\beta=\frac{1}{2}$. The results are depicted in Figure 2.

For backward Euler, the whole solution is free of spurious oscillations, but is strongly damped after each impact. The energy decreases to reach approximately $20 \%$ of its initial value after the fourth impact. This matches with the theory (see Cor. 3.2).

For the most diffusive variant of Newmark $\left(\gamma=1, \beta=\frac{1}{2}\right)$, the displacement and the contact pressure are free of spurious oscillations, and are strongly damped, but not as much as for backward Euler. The energy decreases strongly and reaches at the end approximately $40 \%$ of its initial value. This illustrates as well the theoretical stability study (see Cor. 3.5).

For the variant of Newmark with $\gamma=\beta=\frac{1}{2}$, the displacement is only slightly damped, and the energy is almost conserved, but some spurious oscillations are observed in the contact pressure and in the energy. Note 

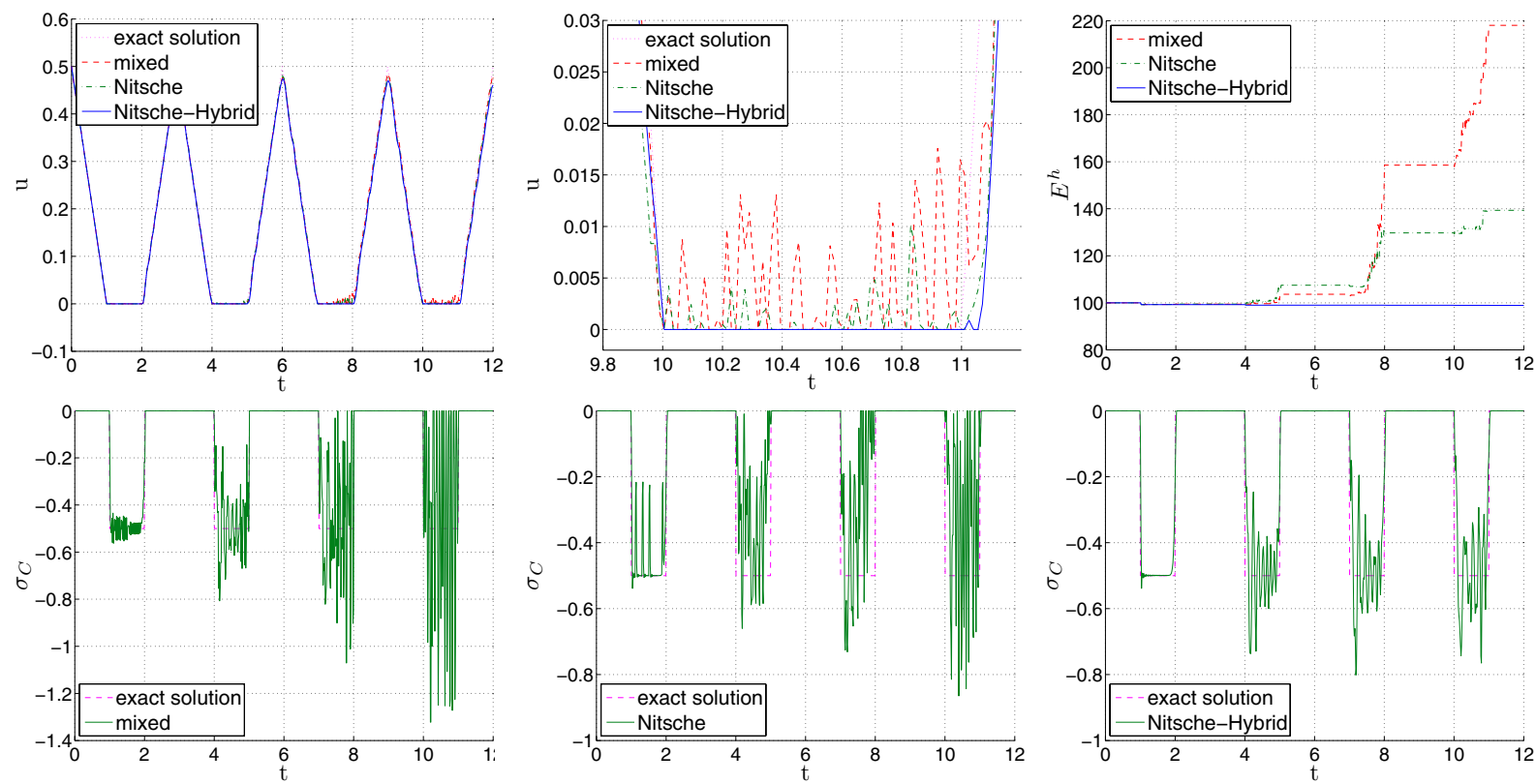

Figure 3. Multiple impacts. Comparison of mixed, Nitsche and Nitsche-Hybrid methods. Displacement $u$ (top left), zoom on displacement $u$ during the fourth impact (top center), energy $E^{h}$ (top right) and contact pressure $\sigma_{C}$ at midpoint (bottom).

the interest of this variant, which, among the family of Newmark schemes, is the most diffusive that remains of order 2. Compared with the variant of order $1\left(\gamma=1, \beta=\frac{1}{2}\right)$, it introduces only a very small amount of numerical dissipation and seems to remain stable.

The augmented energy $E_{\Theta}^{h}$ is not displayed, but is almost equal to the mechanical energy $E^{h}$ in this case (due to the factor $\gamma_{h}$, of the order of $10^{-8}$, in the term $R^{h}$ ).

\subsubsection{Behaviour of (almost) conservative schemes}

We now consider two time-marching schemes that keep the energy constant in the linear regime: the Newmark scheme (2.7) with parameters $\gamma=0.5$ and $\beta=0.25$ ("Nitsche" in the figures), and the Hybrid scheme (2.8) ("Nitsche-Hybrid" in the figures). Recall that, with these parameters, the Newmark scheme (or equivalently the $\theta$-scheme with $\theta=0.5$ ) corresponds to the well-known Crank-Nicolson scheme. Moreover we make comparisons between semi-discretizations with Nitsche and with standard (mixed) method (see, e.g., [28, 29]) ("mixed" in the figures). For the mixed method, we also discretize in time with Newmark, and $\gamma=0.5, \beta=0.25$. We first investigate the symmetric variant $\Theta=1$ with a small parameter $\gamma_{0}=10^{-6}$. The results are depicted in Figure 3 .

The first observation is that for these parameters, Nitsche's method combined to Crank-Nicolson timediscretization behaves quite similarly as the mixed method. In particular, strong increasing spurious oscillations on the contact stress are observed, as well as small increasing spurious oscillations on the displacement. Note that for the contact stress, we plotted the value $\sigma_{C}^{n+\frac{1}{2}}$, i.e., the value at midpoint (though no strong difference with $\sigma_{C}^{n}$ has been observed). The energy is not conserved and sharply grows. We observe that in comparison to the mixed method, there are smaller amplitude oscillations on the contact stress and a smaller increase of the energy for Nitsche's method. This is simply due to the value of $\gamma_{0}$ : if we set $\gamma_{0}$ smaller we recover nearly the same values as in the mixed method. 

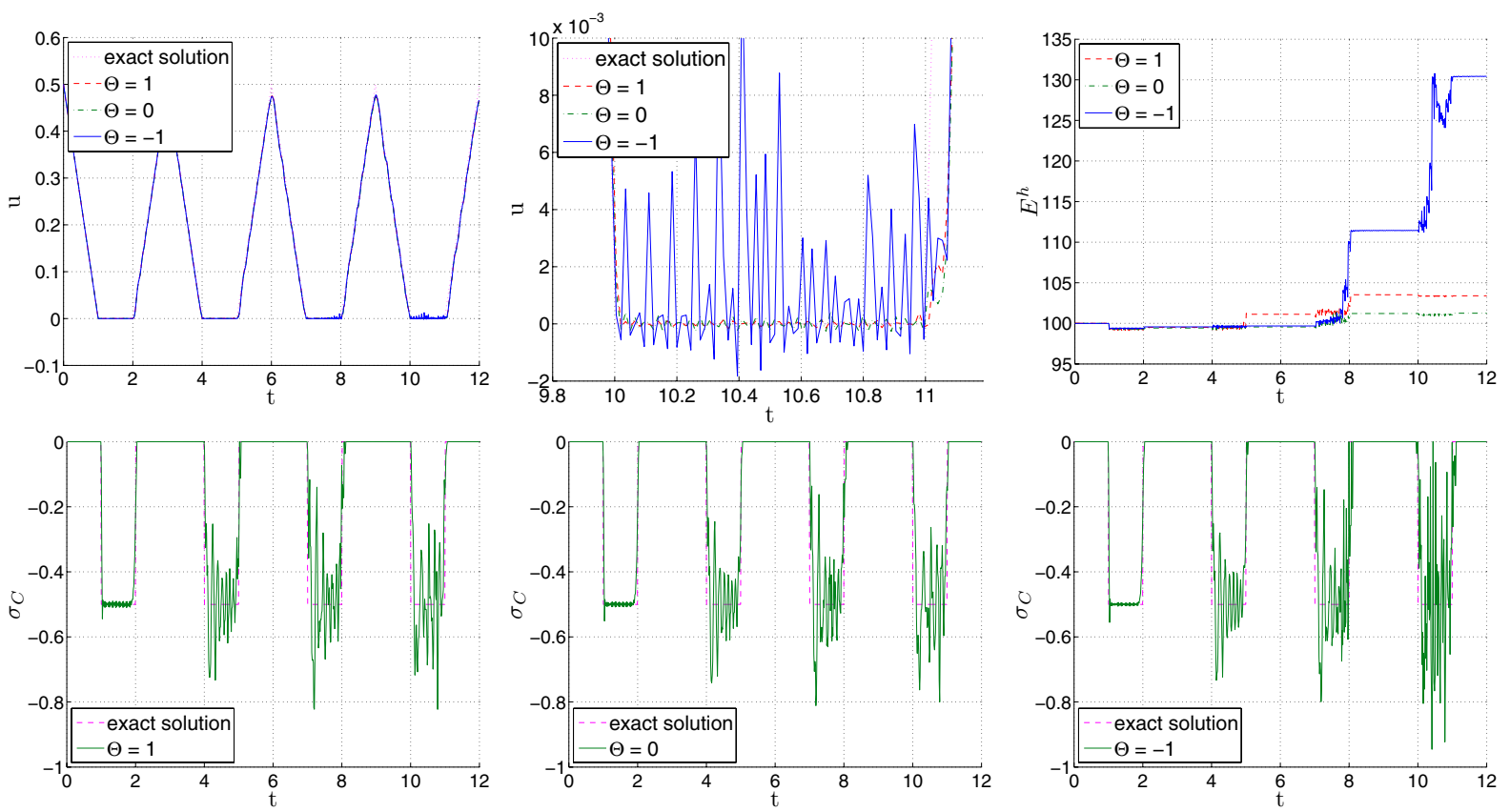

Figure 4. Multiple impacts. Nitsche with $\gamma_{0}=0.1$, and $\Theta=1,0,-1$. Displacement $u$ (top left), zoom on displacement $u$ during the fourth impact (top center), energy $E^{h}$ (top right) and contact pressure $\sigma_{C}$ at midpoint (bottom).

Conversely, for Nitsche's method combined to the Hybrid time-marching scheme, the spurious oscillations on the displacement disappear. Some spurious oscillations are still present on the contact stress, but the amplitude is lower than for mixed and Nitsche's methods. The energy is almost preserved: as predicted by the theory (Cor. 3.8) it decreases slightly, of approximately $1 \%$.

The augmented energy $E_{\Theta}^{h}$ is not plotted, since in this example it has almost the same value as the energy $E^{h}$ (the magnitude of $R^{h}$ is approximately $10^{-8}$ ).

\subsubsection{Influence of Nitsche's parameters}

In this part we study the influence of Nitsche's parameters $\Theta$ and $\gamma_{0}$. For a small $\gamma_{0}\left(=10^{-6}\right)$, the numerical behaviour remains the same whatever is the value of $\Theta=-1,0,1$. In particular, for Nitsche we recover the same curves as in Figure 3, with spurious oscillations on the displacement and the contact stress, as well as a significant increase of the energy. At the opposite, for the Nitsche-Hybrid scheme, the displacement remains free of spurious oscillations and the energy loss is the same as in the symmetric case (approximately 1\% of energy loss). Some spurious oscillations remain on the contact stress, but are of smaller amplitude in comparison with mixed and Nitsche's methods.

For a moderate $\gamma_{0}(=0.1)$, we compare also the values $\Theta=-1,0,1$ as well as Nitsche and Nitsche-Hybrid. The results are depicted in Figure 4 for Nitsche's scheme. Small spurious oscillations remain on the displacement, as well as significant spurious oscillations on the contact pressure. These oscillations are larger for $\Theta=-1$. The energy increase is only of a few percents (approximately 2 to $4 \%$ ) for $\Theta=0,1$ and of nearly $30 \%$ for $\Theta=-1$. As a result, increasing $\gamma_{0}$ permits to reduce the spurious oscillations and to moderate the energy increase. This effect is stronger for $\Theta=0,1$ than for the skew-symmetric method $\Theta=-1$.

The augmented energy $E_{\Theta}^{h}$ is almost equal to the energy $E^{h}$, despite the increased value of $\gamma_{0}$ (the magnitude of $R^{h}$ is of approximately $\left.10^{-4}\right)$. Consequently it is not displayed here. 

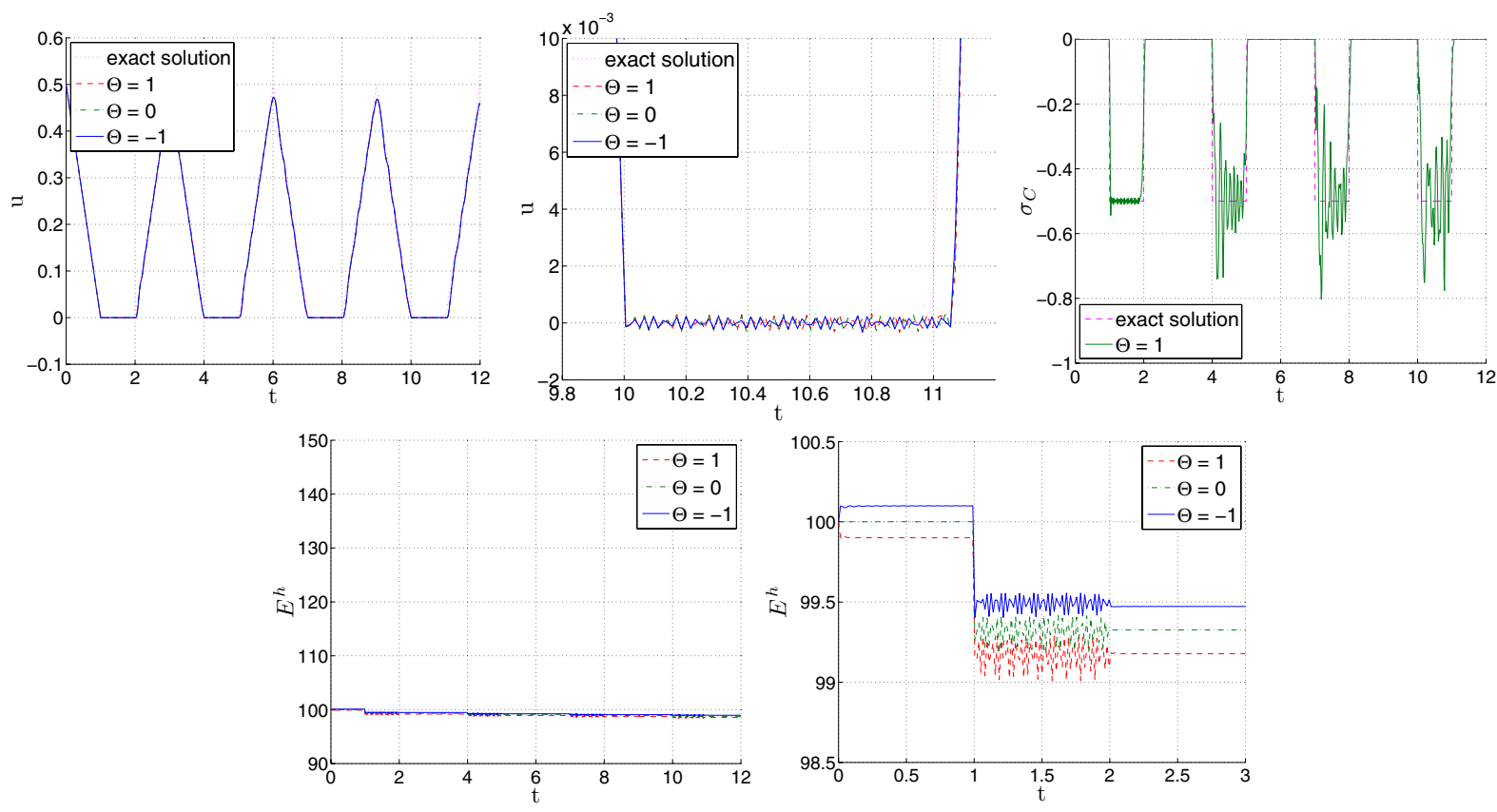

Figure 5. Multiple impacts. Nitsche-Hybrid with $\gamma_{0}=0.1$, and $\Theta=1,0,-1$. Displacement $u$ (top left), zoom on displacement $u$ during the fourth impact (top center), contact pressure $\sigma_{C}$ at midpoint (top right), energy $E^{h}$ (bottom left), zoom on the energy during the first impact (bottom right).

For the Hybrid scheme, the results are depicted in Figure 5. For the Nitsche-Hybrid scheme, the difference between $\gamma_{0}=10^{-6}$ and $\gamma_{0}=0.1$ is less perceptible than for Nitsche. Note however that, for all values of $\Theta$, there are some low amplitude oscillations on the displacement, which are not present when $\gamma_{0}=10^{-6}$. There are still some spurious oscillations on the contact pressure (the curves for $\Theta=0$ and $\Theta=-1$ are not displayed, but are almost the same as the displayed curve for $\Theta=1$ ). The energy curves for different values of $\Theta$ are not superimposed any longer and they present small oscillations during each contact phase, that are not visible when $\gamma_{0}=10^{-6}$. The energy is still approximately conserved, with a dissipation of a few percents. Once more, the curves for the augmented energy $E_{\Theta}^{h}$ are the same as for the energy $E^{h}$.

For a high $\gamma_{0}(=1)$, we still compare the values $\Theta=-1,0,1$ as well as Nitsche and Nitsche-Hybrid. The results are depicted in Figures 6 and 7 for Nitsche.

First, for $\Theta=1$ the results are quite unphysical, and are not displayed, except the energy: after having reached the rigid support, the bar still continues to extend. The energy $E^{h}$ increases monotonically up to an extremely high value, while the augmented energy $E_{\Theta}^{h}$ remains almost constant. For the non-symmetric methods $\Theta=-1,0$, the results are comparable and close to those of $\gamma_{0}=0.1$ : the spurious oscillations on the displacement and on the contact pressure are more or less of the same magnitude, and the energy increases only of $3 \%$ approximately. For $\Theta=-1,0$, the augmented energy $E_{\Theta}^{h}$ remains nearly identical as $E^{h}$, in contrast with the case $\Theta=1$.

When $\gamma_{0}$ becomes greater than 1 , the Newton solver fails to converge in the symmetric case $\Theta=1$. For $\Theta=0$, when $\gamma_{0}$ is of the order of 10 or 100 , the energy is not conserved anymore and low-frequency spurious oscillations appear in the solution. For $\Theta=-1$, and $\gamma_{0}=10,100$, the results are the same as for $\gamma_{0}=1$, and the solution deteriorates only when $\gamma_{0}$ is very high, of the order of $10^{4}$.

The results are then depicted in Figure 8 for Nitsche-Hybrid. For $\Theta=1$, despite the high value of $\gamma_{0}$, we 

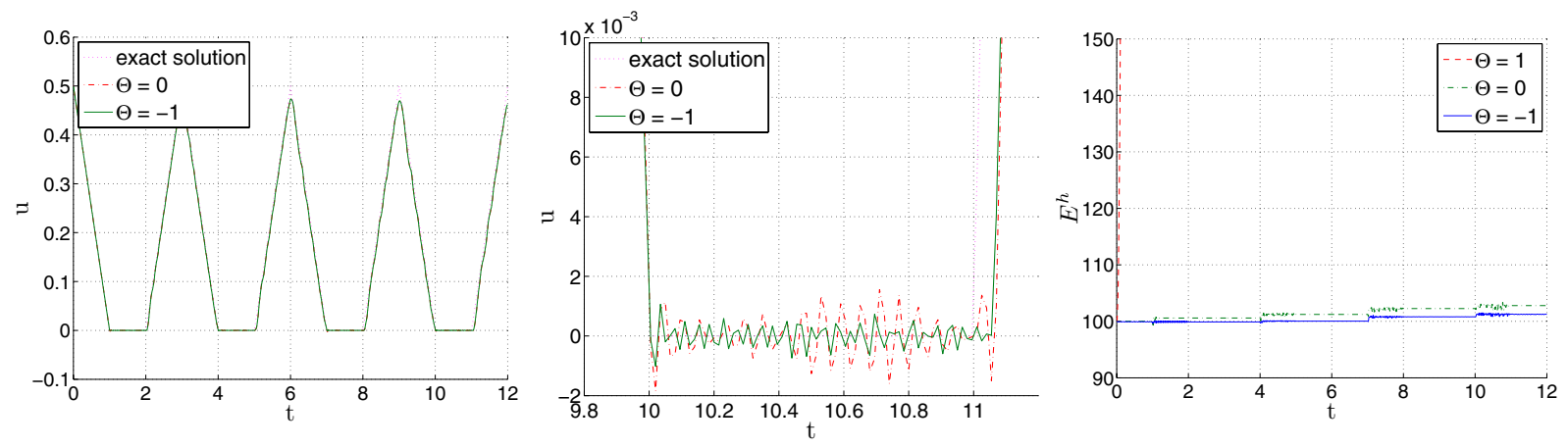

Figure 6. Multiple impacts. Nitsche with $\gamma_{0}=1$, and $\Theta=1,0,-1$. Displacement $u$ (left), zoom on displacement $u$ during the fourth impact (center) and energy $E^{h}$ (right).
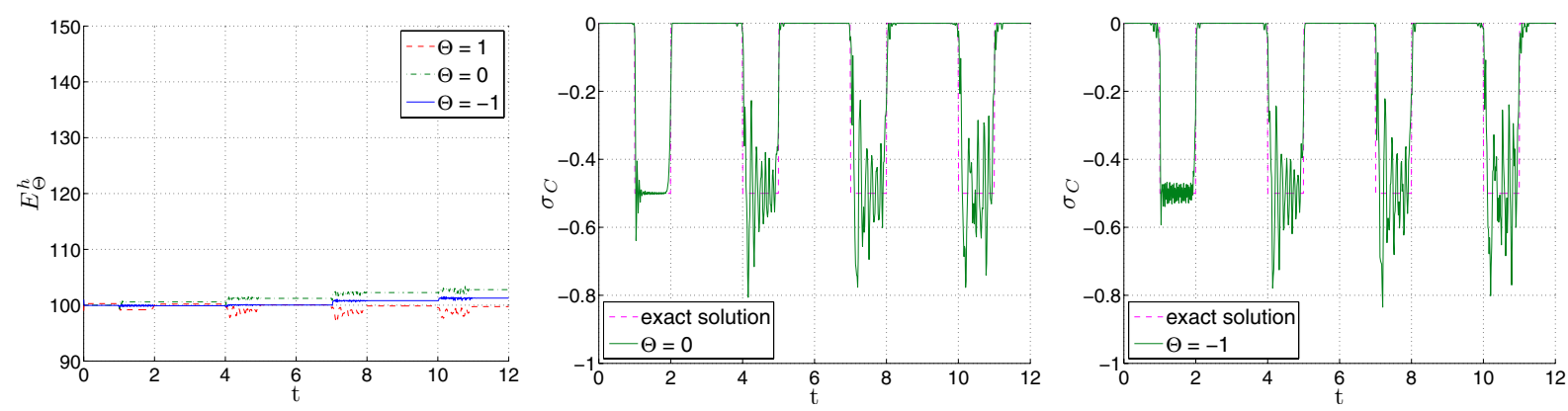

Figure 7. Multiple impacts. Nitsche with $\gamma_{0}=1$, and $\Theta=1,0,-1$. Augmented energy $E_{\Theta}^{h}$ (left) and contact pressure $\sigma_{C}$ at midpoint (center and right).

still obtain a solution but that is unphysical: the sign of displacement and velocity are opposite respectively to the analytical solution, and the energies $E^{h}$ and $E_{\Theta}^{h}$ increase significantly during each impact, up to $10^{4}$ times their initial values. These results are not displayed. For $\Theta=-1,0$ the spurious oscillations on the displacement and contact pressure are slightly greater than for small or moderate values of $\gamma_{0}$. The energies $E^{h}$ and $E_{\Theta}^{h}$ are not preserved any longer, but their increase is small, less than $3 \%$.

In the symmetric case $\Theta=1$, for values of $\gamma_{0}$ greater than 1, the Newton algorithm fails to converge. For $\Theta=0$, the same behaviour as for Nitsche is observed: when $\gamma_{0}$ is higher than 1 , low-frequency spurious oscillations appear on the solution and the energy is not preserved. For $\Theta=-1$, increasing $\gamma_{0}$ above 1 results in an unphysical solution with a strong energy increase (but the Newton algorithm still converges).

Finally, remark that the behaviour of the symmetric scheme $\Theta=1$ is in agreement with the theory (see [5], Props. 3.5 and 3.6) which predicts that well-posedness requires $\gamma_{0}$ small in this case.

\subsubsection{Comparison with modified mass method}

We compare our results to those obtained with the modified mass method (see, e.g., [19]). The chosen method to compute the modified mass matrix is the simplest possible, since we set the entries associated with the contact node to 0 and no mass redistribution is considered (see also [10]). We combine the modified mass method either to the standard (mixed) method or to Nitsche's method for the treatment of contact conditions. The chosen time-marching scheme is still Crank-Nicolson (Newmark with $\gamma=\frac{1}{2}, \beta=\frac{1}{4}$ ). For Nitsche's semi-discretization, 

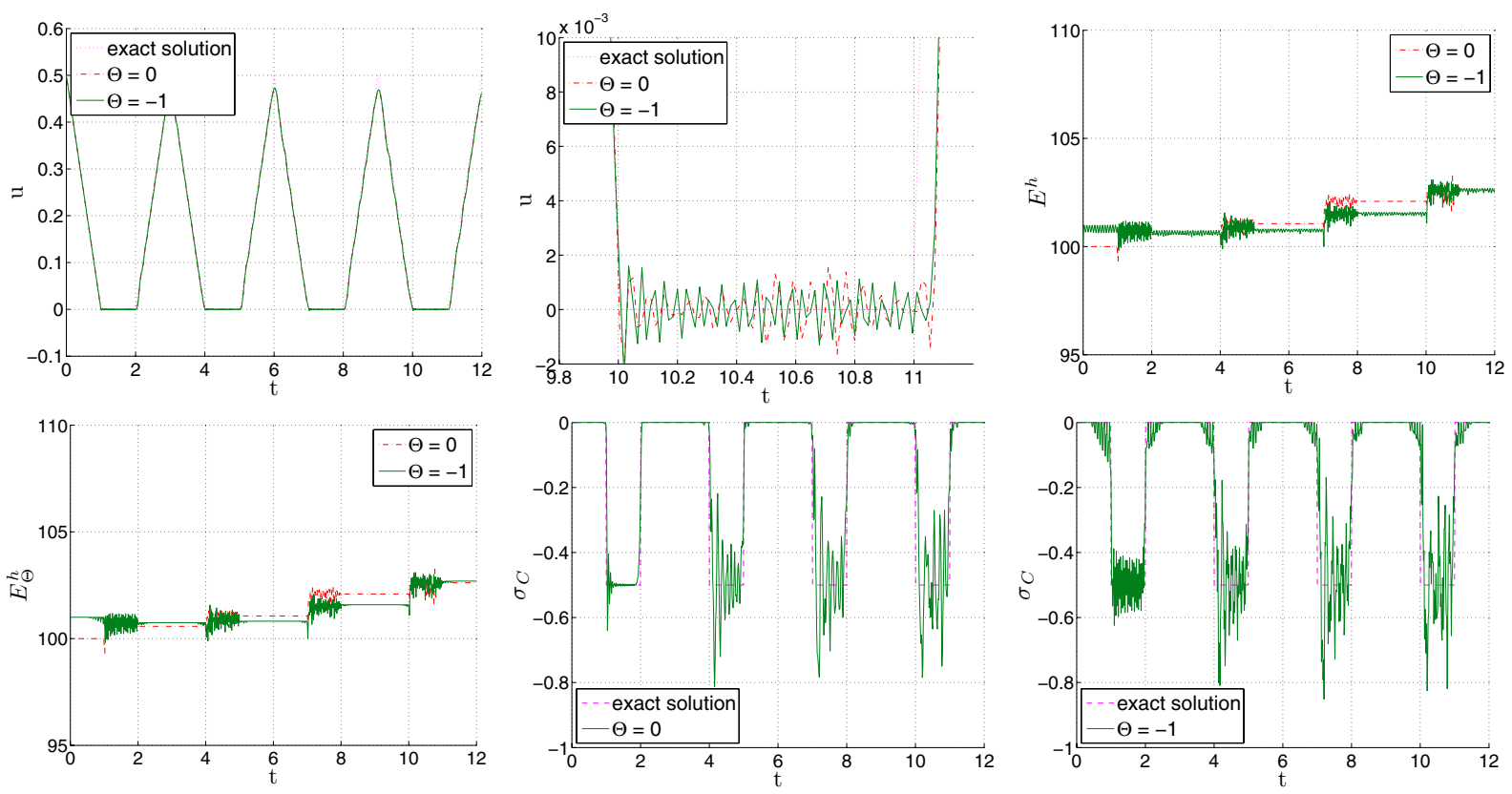

Figure 8. Multiple impacts. Nitsche-Hybrid with $\gamma_{0}=1$, and $\Theta=0,-1$. Displacement $u$ (top left), zoom on displacement $u$ during the fourth impact (top center), energies $E^{h}$ (top right) and $E_{\Theta}^{h}$ (bottom left), and contact pressure $\sigma_{C}$ at midpoint (bottom center and right).

we set $\Theta=1$ and $\gamma_{0}=10^{-6}$. These two methods are compared to the Nitsche-Hybrid scheme, with the same parameters and without modified mass. The results are depicted in Figure 9.

The three methods compare well and there is no significant difference: the displacement is free of spurious oscillations, the energy is quite well preserved, with only 1 or $2 \%$ of dissipation and some small spurious oscillations are still present on the contact pressure, that are of similar magnitude.

This behaviour is well-known for modified mass combined to mixed discretization of the contact (see, e.g., $[10,19])$. These results show that, also for Nitsche's discretization of the contact condition, the modified mass improves the quality of the solution in terms of spurious oscillations and energy conservation. In this test-case, the treatment through modified mass produces almost the same effects as the Hybrid time-marching scheme.

\subsubsection{Influence of space/time discretization parameters}

We study the convergence towards the exact solution when space and time discretization parameters $h$ and $\tau$ tend to 0 . We investigate various space semi-discretizations: mixed, Nitsche (with $\Theta=1$ and $\gamma_{0}=10^{-6}$ ) and modified mass method. These are combined either with the Crank-Nicolson scheme or with the Hybrid scheme (for Nitsche). The curves are obtained by setting $h=\tau$, so the Courant number remains constant: $\nu_{C}=1$. We start with the values $h=\tau=1$ and, each time, we divide the previous values by a factor 4 to refine the discretization.

In order to compute the error curves on the displacement $u$, we introduce the following notations (see [12]): let

$$
u^{h \tau}:=\left(u^{h, 0}, \ldots, u^{h, N}\right) \in\left(V^{h}\right)^{N+1}
$$

be the collection of all the fully discrete solutions for all time steps $t^{0}, \ldots, t^{N}$. Let

$$
u^{\tau}:=\left(u\left(t^{0}\right), \ldots, u\left(t^{N}\right)\right) \in V^{N+1}
$$



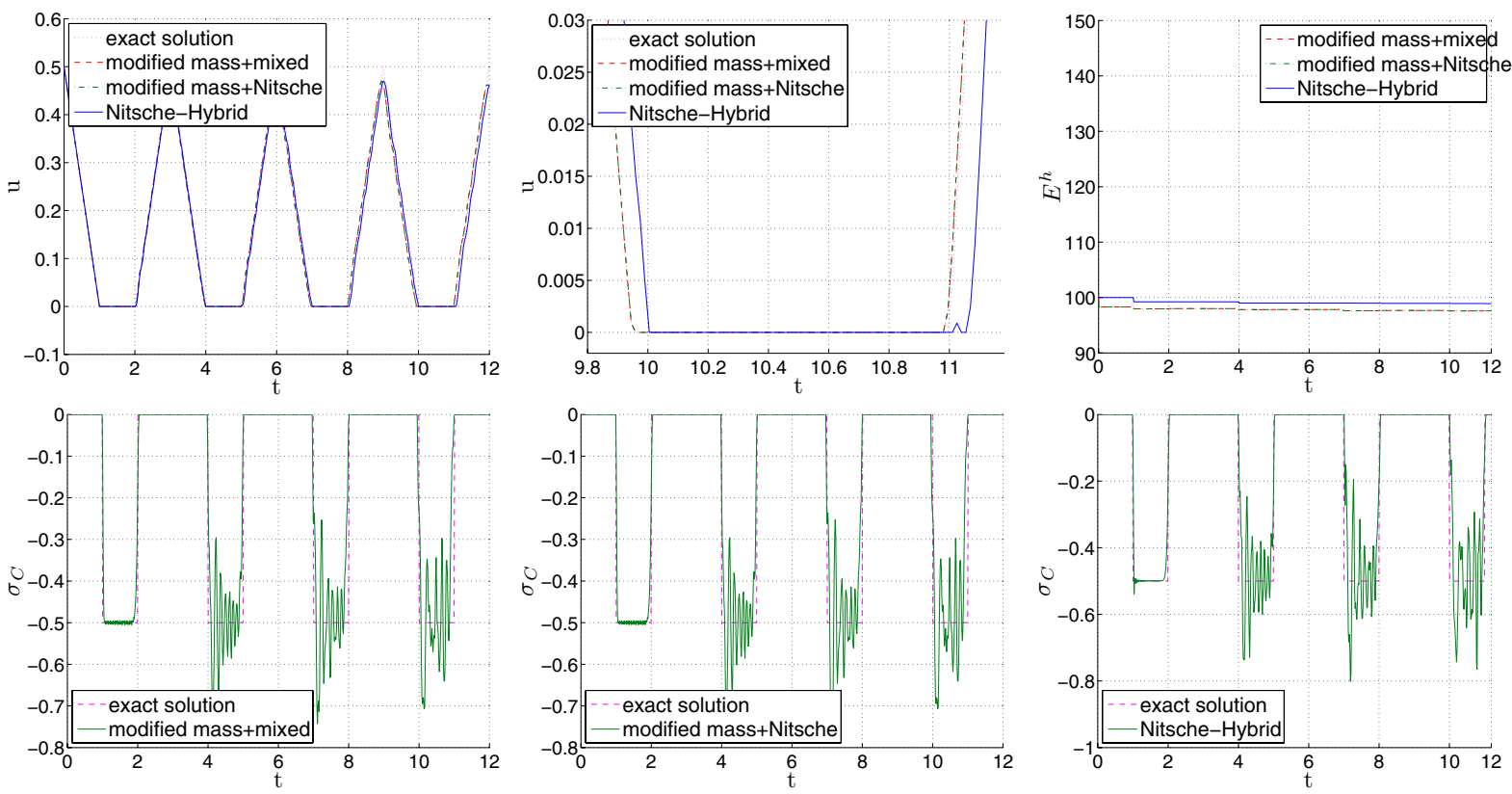

FIgURE 9. Multiple impacts. Comparison of mixed with modified mass, Nitsche with modified mass and Nitsche-Hybrid methods. Displacement $u$ (top left), zoom on displacement $u$ during the fourth impact (top center), discrete energy $E^{h}$ (top right) and contact pressure $\sigma_{C}$ at midpoint (bottom).

be the collection of all the snapshots of the exact solution for all time steps $t^{0}, \ldots, t^{N}$. We define the errors $e^{h \tau}:=u^{h \tau}-u^{\tau}$ and $e^{h, n}:=u^{h, n}-u\left(t^{n}\right)$ for all $n=0, \ldots, N$ (so that $e^{h \tau}=\left(e^{h, 0}, \ldots, e^{h, N}\right)$ ). We will make use of the following discrete counterparts of the $L^{2}\left(0, T ; L^{2}(\Omega)\right)$ and $L^{2}\left(0, T ; H^{1}(\Omega)\right)$ norms:

$$
\left\|e^{h \tau}\right\|_{l^{2}\left(\tau ; L^{2}(\Omega)\right)}:=\left(\tau \sum_{n=0}^{N}\left\|e^{h, n}\right\|_{0, \Omega}^{2}\right)^{\frac{1}{2}}, \quad\left\|e^{h \tau}\right\|_{l^{2}\left(\tau ; H^{1}(\Omega)\right)}:=\left(\tau \sum_{n=0}^{N}\left\|e^{h, n}\right\|_{1, \Omega}^{2}\right)^{\frac{1}{2}} .
$$

We also compute error curves on the contact pressure $\sigma_{C}$ at midpoint, and on the energy $E$. These errors, noted respectively $e_{\sigma}^{h \tau}$ and $e_{E}^{h \tau}$, are computed in the $l^{2}(\tau)$ norm which is defined as follows for a scalar (finite) sequel $x^{\tau}:=\left(x^{n}\right)_{n=0, \ldots, N}:\left\|x^{\tau}\right\|_{l^{2}(\tau)}:=\left(\tau \sum_{n=0}^{N}\left(x^{n}\right)^{2}\right)^{\frac{1}{2}}$. The error curves are depicted in Figure 10.

We observe that all the methods converge in the $l^{2}\left(\tau ; L^{2}(\Omega)\right)$ norm, but that the convergence is slower for the standard mixed and Nitsche methods. The Nitsche-Hybrid and modified mass methods converge better. The error is smaller for the Nitsche-Hybrid scheme but the convergence rate is slightly faster for the modified mass.

In the $l^{2}\left(\tau ; H^{1}(\Omega)\right)$ norm, the mixed and Nitsche methods fail to converge. This is related to the presence of small spurious oscillations on the displacement $u$. On the contrary the Nitsche-Hybrid and modified mass methods converge in this norm. Once again, the error is smaller for Nitsche-Hybrid at the beginning, but the slope is higher for the modified mass so that both curves coincide for the smallest value of $\tau$. It can be noticed that the convergence for the Nitsche-Hybrid scheme is quite slow.

For mixed and Nitsche methods, the approximation of the contact pressure $\sigma_{C}$ does not seem to be improved when $h=\tau$ become smaller, and the slope of the curves is near 0 . Instead, convergence is observed for both 

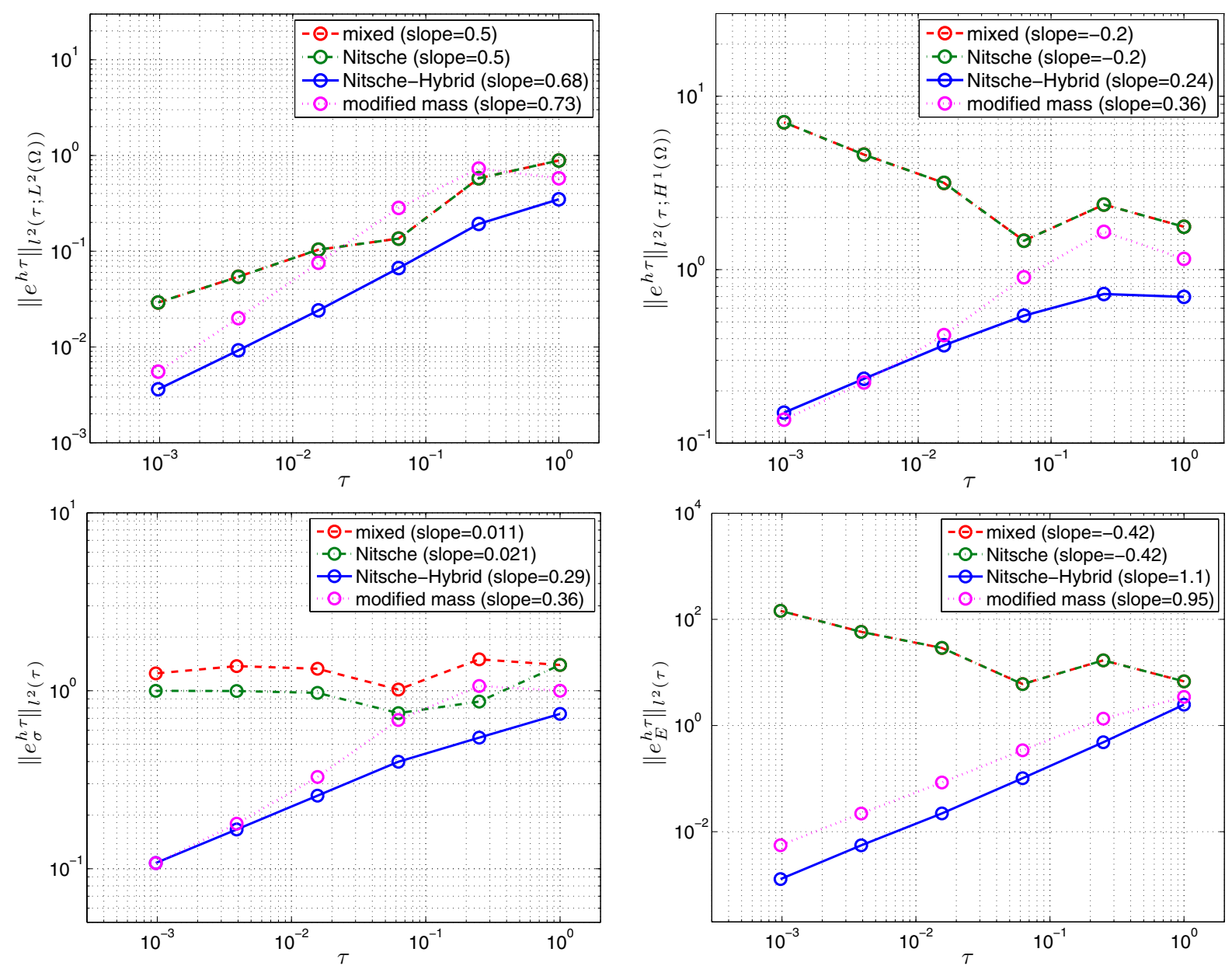

Figure 10. Multiple impacts. Error curves for the displacement $u$ : error $e^{\tau h}$ in norm $l^{2}\left(\tau ; L^{2}(\Omega)\right)$ (top left) and $l^{2}\left(\tau ; H^{1}(\Omega)\right)$ (top right). Error curves for the contact pressure $\sigma_{C}$ (bottom left) and for the energy $E$ (bottom right). Ratio $\tau / h$ is kept constant.

Nitsche-Hybrid and modified mass methods. Once again, the error is smaller at the beginning for NitscheHybrid, but convergence is faster for the modified mass.

Eventually we recover the fact that mixed and Nitsche methods (combined to Crank-Nicolson) do not preserve the energy. If discretization parameters are taken smaller, the result is worse in the sense that the energy blows up and there is no convergence to a solution which preserves the energy. At the opposite, and quite in agreement with theoretical results (see Cor. 3.8 and, e.g., [19]), Nitsche-Hybrid and modified mass methods conserve almost the energy, and the conservation is better when the parameters $h$ and $\tau$ are taken smaller. The Nitsche-Hybrid method provides a smaller error and converges slightly faster than the modified mass method.

\section{2. $2 \mathrm{D} / 3 \mathrm{D}$ numerical experiments: multiple impacts of a disc/a sphere}

Numerical experiments are then carried out in 2D, to assess the behaviour of Nitsche's method in a more realistic situation. We study the impact of a disc on a rigid support. The physical parameters are the 

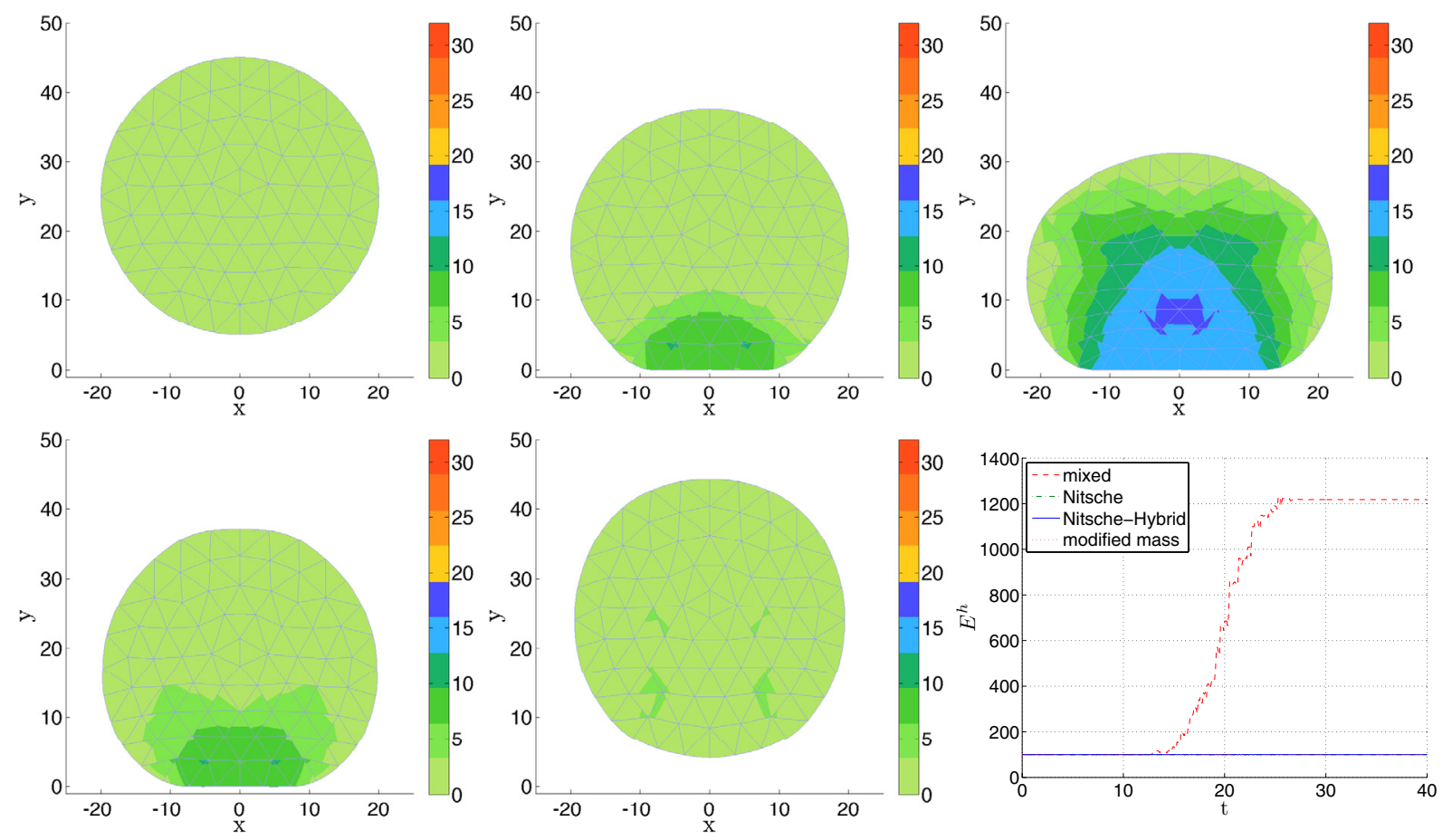

Figure 11. 2D impact of a disc. Deformed configuration and von Mises strain at $t=$ $0,12,18,24,38$, for Nitsche-Hybrid. Energy $E^{h}$ for different methods.

following: the diameter of the disc is $D=40$, the Lamé coefficients are $\lambda=20$ and $\mu=20$, and the material density is $\rho=1$. The total simulation time is $T=40$.

The volume load in the vertical direction is set to $\|\mathbf{f}\|=0.1$ (gravity, oriented towards the support). On the upper part of the boundary an homogeneous Neumann condition $\mathbf{g}=\mathbf{0}$ is applied and the lower part of the boundary is the contact zone $\Gamma_{C}$. There is no initial displacement $\left(\mathbf{u}_{0}=\mathbf{0}\right)$ and no initial velocity $\left(\dot{\mathbf{u}}_{0}=\mathbf{0}\right)$. We introduce an initial gap whose value is 5 . In such a situation, there is to our knowledge no closed-form solution to validate the numerical results.

For space semi-discretization, Lagrange finite elements of order $k=2$ have been used. The mesh size is $h=4$. Integrals of the non-linear term on $\Gamma_{C}$ are computed with standard quadrature formulas of order 4 .

We carry out some tests, with a time-step $\tau=0.1$. The Nitsche parameters are $\Theta=1, \gamma_{0}=0.001$. The results are depicted in Figure 11.

Once again, we compare mixed and Nitsche's semi-discretization combined with Crank-Nicolson, the NitscheHybrid method and the modified mass method (still with Crank-Nicolson). The deformation is depicted only for the Nitsche-Hybrid method, as similar results are obtained with Nitsche and modified mass methods.

For Nitsche-Hybrid, the deformation presents no spurious oscillations and the energy $E^{h}$ is almost preserved, in agreement with the theory and the numerical experiments in 1D. Of course the same observation applies for the modified mass method. Also, for Nitsche, the results are very similar to those observed with Nitsche-Hybrid. This is due to the moderate value of $\gamma_{0}$, as it was already observed in the $1 \mathrm{D}$ case. The choice of this value is motivated by generalized Newton's algorithm, which has difficulties to converge whenever $\gamma_{0}$ is too small (see [27]). Finally, for the mixed discretization, we recover that the energy is not preserved, and some spurious oscillations appear in the strain (not displayed). 

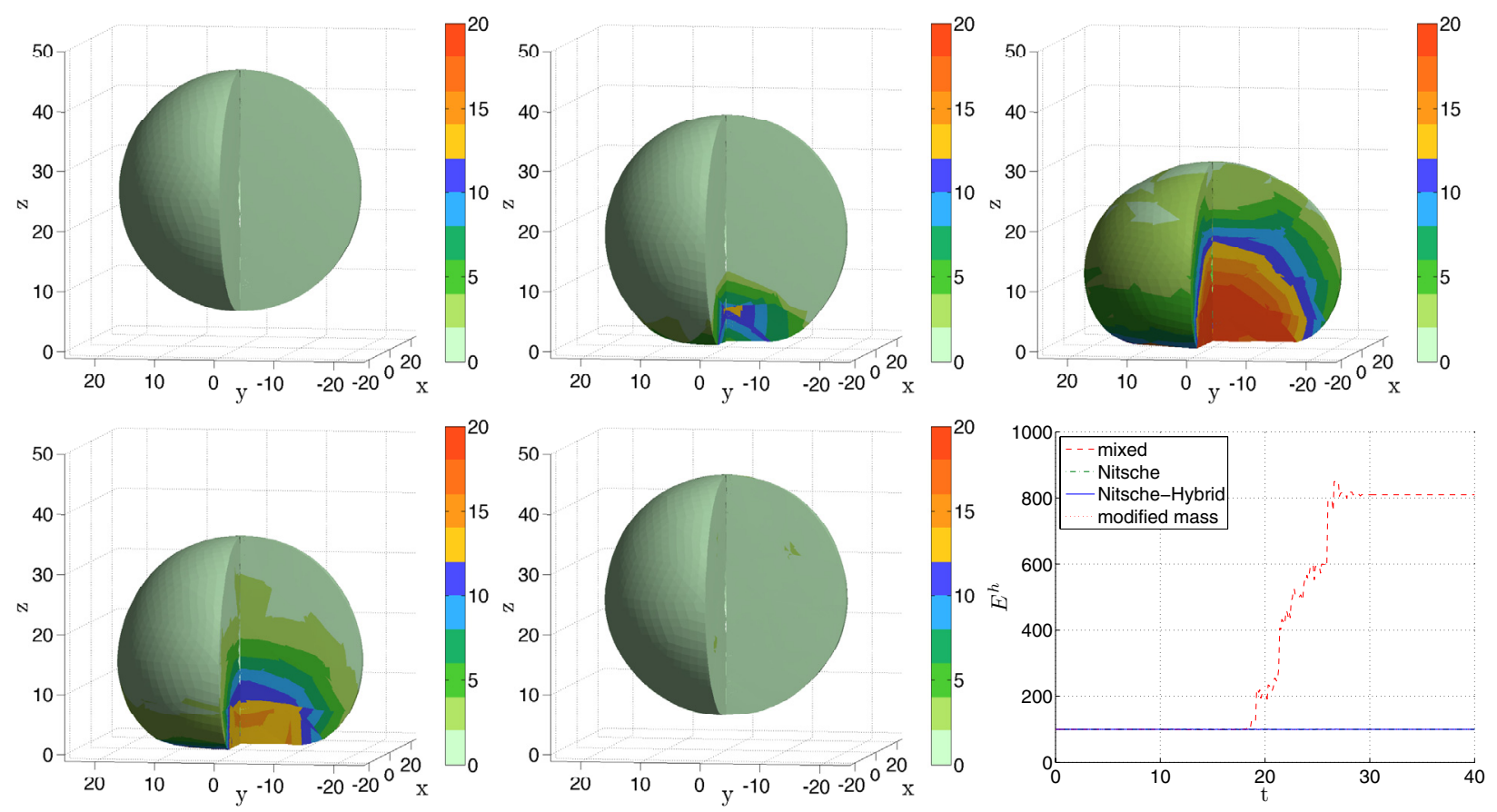

Figure 12. 3D impact of a sphere. Deformed configuration and von Mises strain at $t=$ $0,12,18,24,38$, for Nitsche-Hybrid. Energy $E^{h}$ for different methods.

A similar experiment is carried out in $3 \mathrm{D}$. The parameters are exactly the same as in $2 \mathrm{D}$, except for the mesh size $h \simeq 8$ (400 elements are used). The results are depicted in Figure 12 .

The same conclusions hold as in the 2D case. In particular Nitsche, Nitsche-Hybrid and modified mass methods preserve quite well the energy, conversely to the mixed method.

\section{Conclusion AND PERSpectives}

By itself, the Nitsche-based treatment of contact conditions in elastodynamics does not cure the whole range of numerical problems that arise from other discretizations, such as the classical mixed method. Though for space semi-discretization, Nitsche's method permits to recover a well-posed problem and conservation of an augmented energy when $\Theta=1$, these properties are lost when a standard conservative scheme such as CrankNicolson is applied for time discretization. Indeed, a term in the energy estimates remains that allows creation of artificial energy (see for instance Rem. 3.3). This is observed in practice as well: the energy may increase significantly at each impact, and it is accompanied by spurious oscillations both on the contact pressure and on the displacement.

One first remedy is to combine Nitsche and modified mass methods, and then the energy is quite well preserved and, at least, the displacement is free of spurious oscillations. The resulting scheme behaves as the mixed method combined to modified mass, with the only difference (and maybe advantage) that it remains a primal formulation (no Lagrange multiplier is needed).

Another possibility is to apply the Hybrid time-marching scheme, in which the contact term may be discretized with either Crank-Nicolson or Midpoint when contact conditions are activated. The choice of each alternative is performed in order to prevent creation of artificial energy. This new scheme is slightly dissipative, but the amount of numerical dissipation is very small, and vanishes as $\tau$ becomes smaller. The resulting (fully discrete) 
Nitsche-Hybrid method performs quite as well as the modified mass in terms of energy conservation and of quality of the whole solution.

Note that this Hybrid time-discretization takes advantage of the Nitsche-based formulation, and is not adaptable to a mixed formulation of contact conditions. However it may be suitable for penalized contact. In comparison to modified mass methods, it requires a small additional effort in terms of implementation.

Among the perspectives are the theoretical analysis of convergence of semi-discrete and fully discrete methods, a numerical study of explicit time-marching schemes, and also the design of other well-fitted time-marching schemes.

Acknowledgements. We acknowledge Farshid Dabaghi for having provided Figure 1, and Nabile Boussaïd for some suggestions to improve the presentation. We thank also the anonymous referees for their comments that helped to improve the paper.

\section{REFERENCES}

[1] R.A. Adams, Sobolev spaces. Vol. 65 of Pure Appl. Math. Academic Press, New York, London (1975).

[2] F. Armero and E. Petöcz, Formulation and analysis of conserving algorithms for frictionless dynamic contact/impact problems. Comput. Methods Appl. Mech. Engrg. 158 (1998) 269-300.

[3] Y. Ayyad, M. Barboteu and J.R. Fernández, A frictionless viscoelastodynamic contact problem with energy consistent properties: numerical analysis and computational aspects. Comput. Methods Appl. Mech. Engrg. 198 (2009) 669-679.

[4] N.J. Carpenter, R.L. Taylor and M.G. Katona, Lagrange constraints for transient finite element surface contact. Int. J. Numer. Methods Engrg. 32 (1991) 103-128.

[5] F. Chouly, P. Hild and Y. Renard, A Nitsche finite element method for dynamic contact. 1. Space semi-discretization and time-marching schemes. ESAIM: M2AN 49 (2015) 481-502.

[6] P.G. Ciarlet, Handbook of Numerical Analysis, The finite element method for elliptic problems. Edited by P.G. Ciarlet and J.L. Lions, vol. II, Chap. 1. North Holland (1991) 17-352.

[7] F. Dabaghi, A. Petrov, J. Pousin and Y. Renard, Numerical approximation of a one dimensional elastodynamic contact problem based on mass redistribution method. Submitted (2013). Available at http://hal.archives-ouvertes.fr/hal-00917450.

[8] F. Dabaghi, A. Petrov, J. Pousin and Y. Renard, Convergence of mass redistribution method for the one-dimensional wave equation with a unilateral constraint at the boundary. ESAIM: M2AN 48 (2014) 1147-1169.

[9] P. Deufhard, R. Krause and S. Ertel, A contact-stabilized Newmark method for dynamical contact problems. Int. J. Numer. Methods Engrg. 73 (2008) 1274-1290.

[10] D. Doyen, A. Ern and S. Piperno, Time-integration schemes for the finite element dynamic Signorini problem. SIAM J. Sci. Comput. 33 (2011) 223-249.

[11] Y. Dumont and L. Paoli, Vibrations of a beam between obstacles. Convergence of a fully discretized approximation. ESAIM: M2AN 40 (2006) 705-734.

[12] A. Ern and J.-L. Guermond, Theory and practice of finite elements. In vol. 159 of Appl. Math. Sci. Springer-Verlag, New York (2004).

[13] O. Gonzalez, Exact energy and momentum conserving algorithms for general models in nonlinear elasticity. Comput. Methods Appl. Mech. Engrg. 190 (2000) 1763-1783.

[14] C. Hager, S. Hüeber and B.I. Wohlmuth, A stable energy-conserving approach for frictional contact problems based on quadrature formulas. Int. J. Numer. Methods Engrg. 73 (2008) 205-225.

[15] P. Hauret, Mixed interpretation and extensions of the equivalent mass matrix approach for elastodynamics with contact. Comput. Methods Appl. Mech. Engrg. 199 (2010) 2941-2957.

[16] P. Hauret and P. Le Tallec, Energy-controlling time integration methods for nonlinear elastodynamics and low-velocity impact. Comput. Methods Appl. Mech. Engrg. 195 (2006) 4890-4916.

[17] C. Kane, E.A. Repetto, M. Ortiz and J.E. Marsden, Finite element analysis of nonsmooth contact. Comput. Methods Appl. Mech. Engrg. 180 (1999) 1-26.

[18] H.B. Khenous, Problèmes de contact unilatéral avec frottement de Coulomb en élastostatique et élastodynamique. Etude mathématique et résolution numérique. Ph.D. thesis, INSA de Toulouse (2005).

[19] H.B. Khenous, P. Laborde and Y. Renard, Mass redistribution method for finite element contact problems in elastodynamics. Eur. J. Mech. A Solids 27 (2008) 918-932.

[20] R. Krause and M. Walloth, Presentation and comparison of selected algorithms for dynamic contact based on the Newmark scheme. Appl. Numer. Math. 62 (2012) 1393-1410.

[21] T.A. Laursen and V. Chawla, Design of energy conserving algorithms for frictionless dynamic contact problems. Int. J. Numer. Methods Engrg. 40 (1997) 863-886.

[22] T.A. Laursen and G.R. Love, Improved implicit integrators for transient impact problems - geometric admissibility within the conserving framework. Int. J. Numer. Methods Engrg. 53 (2002) 245-274. 
[23] L. Paoli, Time discretization of vibro-impact. R. Soc. Lond. Philos. Trans. Ser. A Math. Phys. Eng. Sci. 359 (2001) $2405-2428$.

[24] L. Paoli and M. Schatzman, A numerical scheme for impact problems. I. The one-dimensional case. SIAM J. Numer. Anal. 40 (2002) 702-733.

[25] L. Paoli and M. Schatzman, A numerical scheme for impact problems. II. The multidimensional case. SIAM J. Numer. Anal. 40 (2002) 734-768.

[26] Y. Renard, The singular dynamic method for constrained second order hyperbolic equations: application to dynamic contact problems. J. Comput. Appl. Math. 234 (2010) 906-923.

[27] Y. Renard, Generalized Newton's methods for the approximation and resolution of frictional contact problems in elasticity. Comput. Meth. Appl. Mech. Engrg. 256 (2013) 38-55.

[28] B. Wohlmuth, Variationally consistent discretization schemes and numerical algorithms for contact problems. Acta Numerica (2011) 569-734.

[29] P. Wriggers, Computational Contact Mechanics. Wiley (2002). 